\title{
Survey, Identification, and Characterization of Cylindrocarpon-Like Asexual Morphs in Spanish Forest Nurseries
}

Beatriz Mora-Sala, Instituto Agroforestal Mediterráneo, Universitat Politècnica de València, Camino de Vera s/n, 46022-Valencia, Spain; Ana Cabral, Linking Landscape, Environment, Agriculture and Food (LEAF), Instituto Superior de Agronomia, Universidade de Lisboa, Tapada da Ajuda, 1349-017 Lisbon, Portugal; Maela León, Instituto Agroforestal Mediterráneo, Universitat Politècnica de València, Camino de Vera s/n, 46022-Valencia, Spain; Carlos Agustí-Brisach, Departamento de Agronomía, ETSIAM, Universidad de Córdoba, Campus de Rabanales, Edif. C4, 14071 Córdoba, Spain; Josep Armengol and Paloma Abad-Campos, ${ }^{\dagger}$ Instituto Agroforestal Mediterráneo, Universitat Politècnica de València, Camino de Vera s/n, 46022-Valencia, Spain

\begin{abstract}
Cylindrocarpon-like asexual morphs infect herbaceous and woody plants, mainly in agricultural scenarios, but also in forestry systems. The aim of the present study was to characterize a collection of Cylindrocarpon-like isolates recovered from the roots of a broad range of forest hosts from nurseries showing decline by morphological and molecular studies. Between 2009 and 2012, 17 forest nurseries in Spain were surveyed and a total of 103 Cylindrocarpon-like isolates were obtained. Isolates were identified based on DNA sequences of the partial gene regions histone $\mathrm{H} 3$ (his3). For the new species, the internal transcribed spacer and intervening 5.8S nrRNA gene (ITS) region, $\beta$-tubulin (tub2), and translation elongation factor $1-\alpha$ (tefl) were also used to

determine their phylogenetic position. Twelve species belonging to the genera Cylindrodendrum, Dactylonectria, and Ilyonectria were identified from damaged roots of 15 different host genera. The species C. alicantinum, D. macrodidyma, D. novozelandica, D. pauciseptata, D. pinicola, D. torresensis, I. capensis, I. cyclaminicola, I. liriodendri, I. pseudodestructans, I. robusta, and I. rufa were identified. In addition, two Dactylonectria species (D. hispanica sp. nov. and D. valentina $\mathrm{sp}$. nov.), one Ilyonectria species (I. ilicicola sp. nov.), and one Neonectria species ( $N$. quercicola sp. nov.) are newly described. The present study demonstrates the prevalence of this fungal group associated with seedlings of diverse hosts showing decline symptoms in forest nurseries in Spain.
\end{abstract}

Spain has the second largest forest area in the European Union (EU), 27.7 M ha, which accounts for $15.4 \%$ of the total European forest. The most extensive forest systems in Spain include holm oak forests (Quercus ilex) (2.8 M ha, 10.1\% of the forested area), oak range-lands consisting mainly of holm oaks (2.4 M ha), and Aleppo pines (Pinus halepensis) (2 M ha) (MAGRAMA 2014).

Forest systems not only offer market valuable services such as timber, hunting, fishing, and tourism, but also important environmental and social contributions such as carbon capture, hydric and soil regulation, hosting biodiversity, and regulating climate. Climate change has substantially built public awareness about the need of developing forest management strategies to counterbalance the deforestation rate (Trumbore et al. 2015). According to the FAO (2015), in 2010, the deforestation rate in Spain was 57,000 ha per year, while the reforestation rate shed a positive balance with 89,000 ha per year.

Forest nurseries provide woody plants for the afforestation process, but they are also a key point to prevent and control early infections by fungal pathogens, thus guaranteeing the phytosanitary quality of planting materials. An increasing incidence of invasive pathogens related to global tree-planting projects has been reported worldwide (Wingfield et al. 2015). Numerous examples of alien invasions have been reported in the forestry sector, such as: i) Phytophthora cinnamomi root disease or Jarrah dieback; ii) sudden oak death caused by P. ramorum; iii) Dutch elm disease caused by Ophiostoma ulmi; iv) chestnut blight caused by Cryphonectria parasitica; and v) cedar root rot caused by $P$. lateralis (Brasier 2008). Thus, nursery

${ }^{\dagger}$ Corresponding author: P. Abad-Campos; E-mail: pabadcam@eaf.upv.es

Funding: This research was supported by funding from the Spanish project AGL2011-30438-C02-01 (Ministerio de Economía y Competitividad, Spain). It was also funded by Portuguese national funds through Fundação para a Ciência e a Tecnologia grant SFRH/BPD/84508/2012 for Ana Cabral and FCT Unit funding UID/AGR/04129/2013). C. Agustí-Brisach is the holder of a 'Juan de la Cierva-Formación' fellowship from MINECO (Spain).

Accepted for publication 26 March 2018.

(c) 2018 The American Phytopathological Society regulations should be implemented at a global scale and updated phytosanitary measures must be considered as the cornerstone of the all nursery systems (Wingfield et al. 2015).

Cylindrocarpon-like asexual morphs infect herbaceous and woody plants, mainly in agricultural scenarios, but also in forest systems (Agustí-Brisach and Armengol 2013). This group of fungi has been commonly associated with damping-off, root rot, or bark necrosis in forest nurseries and also with cankers on forest stands (Jankowiak et al. 2016). In Swedish conifer nurseries, Cylindrocarpon (Cy.) destructans was the main pathogen isolated from damaged root tissues (Beyer-Ericson et al. 1991). Lilja et al. (1992) reported the presence of $C y$. cylindroides, Cy. destructans, Cy. didymum, Cy. magnusiarum, $C y$. obtusisporum, and $C y$. pineum in seedlings of Pinus (P.) sylvestris and Picea (Pc.) abies in Finnish nurseries. None of these asexual morphs seemed to be pathogenic, but they predisposed $P$. sylvestris to be colonized by the most common saprophytic Cy. destructans. Dumroese and James (2005) stated that in forest and conservation nurseries in the Pacific Northwest of the U.S.A., Cylindrocarpon-like asexual morphs were among the most ubiquitous root pathogens, with $C y$. destructans being the most frequently isolated. Menkis et al. (2006) confirmed the presence of Nectria species ( $N$. gliocladioides, $N$. inventa, $N$. lucida, $N$. macrodydima, and $N$. radicicola) in decayed roots of $P$. sylvestris and $P c$. abies nursery seedlings in Lithuania. In 2009, damping off of $P$. radiata seedlings was observed in a pine nursery in Spain. Dactylonectria (D.) pauciseptata was described as the causal agent of damping-off, extensive root necrosis, and root death, exhibited by the pine seedlings (AgustíBrisach et al. 2011).

In forests, Cylindrocarpon-like asexual morphs have also been reported as the dominant fungi on roots of Fraxinus excelsior, Fagus (F.) sylvatica, and Quercus spp. (Halmschlager and Kowalski 2004; Kubíková 1963; Krzan 1987). Cylindrocarpon-like asexual morphs can hinder the natural regeneration of different tree species. $C y$. destructans arose as the main root pathogen implicated in the absence of regeneration of Taxus baccata and Abies (A.) alba in Poland (Kowalski 1982; Manka et al. 1968). Damping off caused by $C y$. destructans was observed in the natural regeneration of Eucalyptus trees in Australia (Iles et al. 2010; Mwanza and Kellas 1987). In Canada, Axelrood et al. (1998) isolated Cylindrocarpon-like asexual 
morphs from the roots of naturally regenerating seedlings of Pseudotsuga (Ps.) menziesii. Szewczyk and Szwagrzyk (2010) reported that this complex of asexual morphs affected the regeneration of old stands of F. sylvatica and A. alba in Western Carpathians, Poland. In 2012, Neonectria candida (syn. $N$. ramulariae) was described as a pathogen of $F$. crenata seeds in Japan (Hirooka et al. 2012), also affecting its natural regeneration. Jankowiak et al. (2016) characterized a collection of Cylindrocarpon-like fungi associated with beech litter in Austria and Poland and identified five species from $F$. sylvatica and $P$. sylvestris: Ilyonectria crassa, I. pseudodestructans, I. rufa, N. candida, and N. obtusispora, and seven species were identified to genus level (Ilyonectria or Neonectria species).

The taxonomy of Cylindrocarpon-like asexual morphs has been revised several times since the genus Cylindrocarpon was first introduced in 1913 by Wollenweber to describe the asexual morphs of the Nectria section Willkomiotes Wollenw., which included species without chlamydospores (Brayford 1993; Halleen et al. 2006). Afterward, in 1917, the genus Cylindrocarpon also embraced species with mycelial chlamydospores in culture, with $C y$. destructans becoming the most important species of this group. Booth (1966) split the genus into four groups based on the presence or absence of microconidia and chlamydospores (Brayford 1993; Halleen et al. 2006). Further studies transferred species of the Nectria group with Cylindrocarpon-like asexual morphs into Neonectria (Brayford et al. 2004; Mantiri et al. 2001; Rossman et al. 1999). In 2004, the new asexual morph genus, Campylocarpon, was described by Halleen et al. (2004). Later, Chaverri et al. (2011) recognized five novel genera within Neonectria based on characters associated with perithecial anatomy and conidial septation: Campylocarpon, Ilyonectria, Neonectria (Cylindrocarpon s.s.), Rugonectria, and Thelonectria. Finally, Lombard et al. (2014) stated that the genus Ilyonectria was paraphyletic, and therefore designating a new genus Dactylonectria to resolve this. At the same time, the genus Cylindrodendrum was shown to form a well-supported monophyletic sister clade to the Ilyonectria clade.

Within the I. destructans complex, 12 new taxa were delineated mainly based on isolates previously describe as Cy. destructans s.l. from a diverse host range (Cabral et al. 2012a). This study comprised isolates of I. liriodendri from Quercus suber (Q. suber); isolates of I. robusta from Quercus sp. and Quercus robur (Q. robur); I. rufa from A. alba, Ps. menziesii, and Pc. glauca; I. pseudodestructans from Quercus sp., and I. europaea from Aesculus hippocastanum.

Dactylonectria species have also been isolated from forest species; D. estremocensis was isolated from Quercus sp. and $P c$. glauca, D. torresensis from A. nordmanniana and Quercus sp. (Cabral et al. 2012b), and D. pinicola from P. laricio (Lombard et al. 2014).

Several Neonectria species have been associated with forest species, which include $N$. coccinea, $N$. ditissima, $N$. faginata, $N$. fuckeliana, N. lugdunensis, N. major, N. neomacrospora, $N$. obtusispora, and N. tsugae (Castlebury et al. 2006; Lombard et al. 2014).

Sánchez et al. (2002) reported high mortality levels of Quercus seedlings ( $Q$. ilex, $Q$. suber, and $Q$. faginea) caused by $C y$. destructans in a nursery in southeastern Spain, but no further studies have explored the occurrence of species with Cylindrocarpon-like asexual morphs associated with root rot and dieback in Spanish forest nurseries. There is a lack of knowledge about the relevance of this group of fungi in forest nurseries. Thus, the aim of the present study was to characterize a large collection of Cylindrocarpon-like isolates recovered from forest nurseries and a broad range of hosts displaying decline symptoms, by means of phenotypical characterization and DNA analysis.

\section{Materials and Methods}

Fungal isolation. Between 2009 and 2012, extensive surveys were conducted in 17 Spanish forest nurseries located in the provinces of Alicante, Castellón, Tarragona, and Valencia (eastern Spain); and León, Logroño, Soria, and Madrid (central-northern Spain). The survey was focused on plants showing symptoms such as wilting, dieback, chlorosis, foliage discoloration, defoliation, growth reduction, and general decline (Fig. 1A-E).
Affected plants showed root rot and loss of the feeder roots with the presence of necrotic lesions. These symptoms led to a reduction of the root biomass and root hairs, diminishing the volume of the root system and its feeder abilities (Fig. 1F, G), which resulted in plant collapse (Fig. 1A-E). At least three plants per symptomatic species were collected in each nursery and transported to the laboratory for fungal isolation. Affected roots were washed under running tap water, surface disinfested for $1 \mathrm{~min}$ in a $1.5 \%$ sodium hypochlorite solution, and washed twice with sterile distilled water. Small pieces of discolored tissues were plated on potato dextrose agar (PDA) (Biokar-Diagnostics, Zac de Ther, France) amended with $0.5 \mathrm{~g} \mathrm{liter}^{-1}$ of streptomycin sulfate (Sigma-Aldrich, St. Louis, MO, U.S.A.) (PDAS). Plates were incubated for 5 to 10 days at $25^{\circ} \mathrm{C}$ in darkness.

According to morphological characters (mycelium aspect and colony color), 103 isolates of Cylindrocarpon-like asexual morphs representative of different hosts and geographical origins were selected for further analysis (Table 1). These isolates were single-spored with the serial dilution method prior to morphological and molecular characterization (Dhingra and Sinclair 1995). For long-term storage, agar plugs with mycelium and conidia from cultures were stored in $15 \%$ glycerol solution at $-80^{\circ} \mathrm{C}$ in $1.5 \mathrm{ml}$ cryovials.

Morphological characterization. Single conidial cultures were grown for up to 5 weeks at $20^{\circ} \mathrm{C}$ on synthetic nutrient-poor agar (SNA; Nirenberg 1976) with or without the addition of two $1-\mathrm{cm}^{2}$ pieces of sterile filter paper on the medium surface, PDA, and oatmeal agar (OA; Crous et al. 2009) under continuous nearUV fluorescent light (NUV; 400-315 nm; Philips TL 8W BLB, The Netherlands). To induce perithecia of new species, homothallic and heterothallic crosses (for the cases that there is more than one isolate in the species) were performed as described by Cabral et al. (2012a).

Fungal structures were measured at a 1,000× magnification using a Leica DM2500 and images were captured using a Leica DFC295 digital camera with the Leica Application Suite (LAS) version 3.3.0. For this purpose, an agar square was removed and placed on a microscope slide, to which a drop of water was added and overlaid with a cover slip. For each isolate, 30 measurements were obtained for each informative structure. Measurements were obtained with LAS software and round to the nearest $0.5 \mu \mathrm{m}$. The $95 \%$ confidence intervals were determined and the extremes of the conidial measurements are shown in parentheses. For the other structures, only the extremes are presented.

Culture characteristics (texture, density, color, growth front, transparency, and zonation) were described on PDA and OA after incubation at $20^{\circ} \mathrm{C}$ in the dark for 14 days. Color (surface and reverse) was described using the color charts of Rayner (1970).

Cardinal growth temperatures were assessed by inoculating $90 \mathrm{~mm}$ diameter PDA dishes with a $6 \mathrm{~mm}$ diameter plug cut from the edge of an actively growing colony. Growth was determined after 7 days in two orthogonal directions. Trials were conducted at 5 to $35^{\circ} \mathrm{C}$ with $5^{\circ} \mathrm{C}$ intervals, with three replicates per strain at each temperature.

DNA isolation, sequencing and phylogenetic analysis. For DNA extraction, fungal mycelium from pure cultures grown on PDA for 2 to 3 weeks at $25^{\circ} \mathrm{C}$ in darkness were scraped and ground to a fine powder with liquid nitrogen using a mortar and pestle. Total genomic DNA was extracted using the E.Z.N.A. Plant Miniprep Kit (Omega Bio-tek, Doraville, U.S.A.) following the manufacturer's instructions. DNA was visualized by electrophoresis on $1 \%$ agarose gels stained with REALSAFE (REALSAFE Nucleic Acid Staining Solution 20,000×, Durviz S. L., Valencia, Spain) and stored at $-20^{\circ} \mathrm{C}$.

In order to identify the species involved, partial sequences of the histone $\mathrm{H} 3$ (his3) gene region was amplified according to Cabral et al. (2012a). Six isolates (Cy-FO-3, Cy-FO-45, Cy-FO-133, CyFO-224, Cy-FO-225, and Cy-FO-226) were additionally sequenced for the internal transcribed spacer and intervening 5.8S gene (ITS) region, partial regions of the $\beta$-tubulin (tub2) and translation elongation factor $1-\alpha$ (tefl) genes to better resolve their phylogenetic 
position. PCR amplifications were carried out using $1 \times$ PCR buffer, $2.5 \mathrm{mM} \mathrm{MgCl}_{2}, 0.2 \mathrm{mM}$ of each dNTP, $0.4 \mathrm{mM}$ of each primer, $1 \mathrm{U}$ of Taq polymerase (Canvax Biotech, S.L., Córdoba, Spain), and $1 \mu l$ of template DNA $(20 \mathrm{ng} / \mu \mathrm{l})$. The PCR reaction mix was adjusted to a final volume of $25 \mu \mathrm{l}$ with ultrapure sterile water (Chromasolv Plus, Sigma-Aldrich, Steinheim, Germany). The cycle conditions in a Peltier Thermal Cycler-200 (MJ Research) were: $94^{\circ} \mathrm{C}$ for $3 \mathrm{~min}$, followed by 35 cycles of denaturation at $94^{\circ} \mathrm{C}$ for $30 \mathrm{~s}$, annealing at $55^{\circ} \mathrm{C}$ for $30 \mathrm{~s}$, elongation at $72^{\circ} \mathrm{C}$ for $45 \mathrm{~s}$, and a final extension at $72^{\circ} \mathrm{C}$ for $10 \mathrm{~min}$. Primers used were CYLH3F and CYLH3R (Crous et al. 2004b) for his3, ITS1F (Gardes and Bruns 1993) and ITS4 (White et al. 1990) for ITS, T1 (O’Donnell and Cigelnik 1997) and Bt2b (Glass and Donaldson 1995) for tub2, and CylEF-1 (5'ATGGGTAAGGAVGAVAAGAC-3'; J. Z. Groenewald, unpublished) and CylEF-R2 (Crous et al. 2004b) for tef1. After confirmation by agarose gel electrophoresis, PCR products were sequenced in
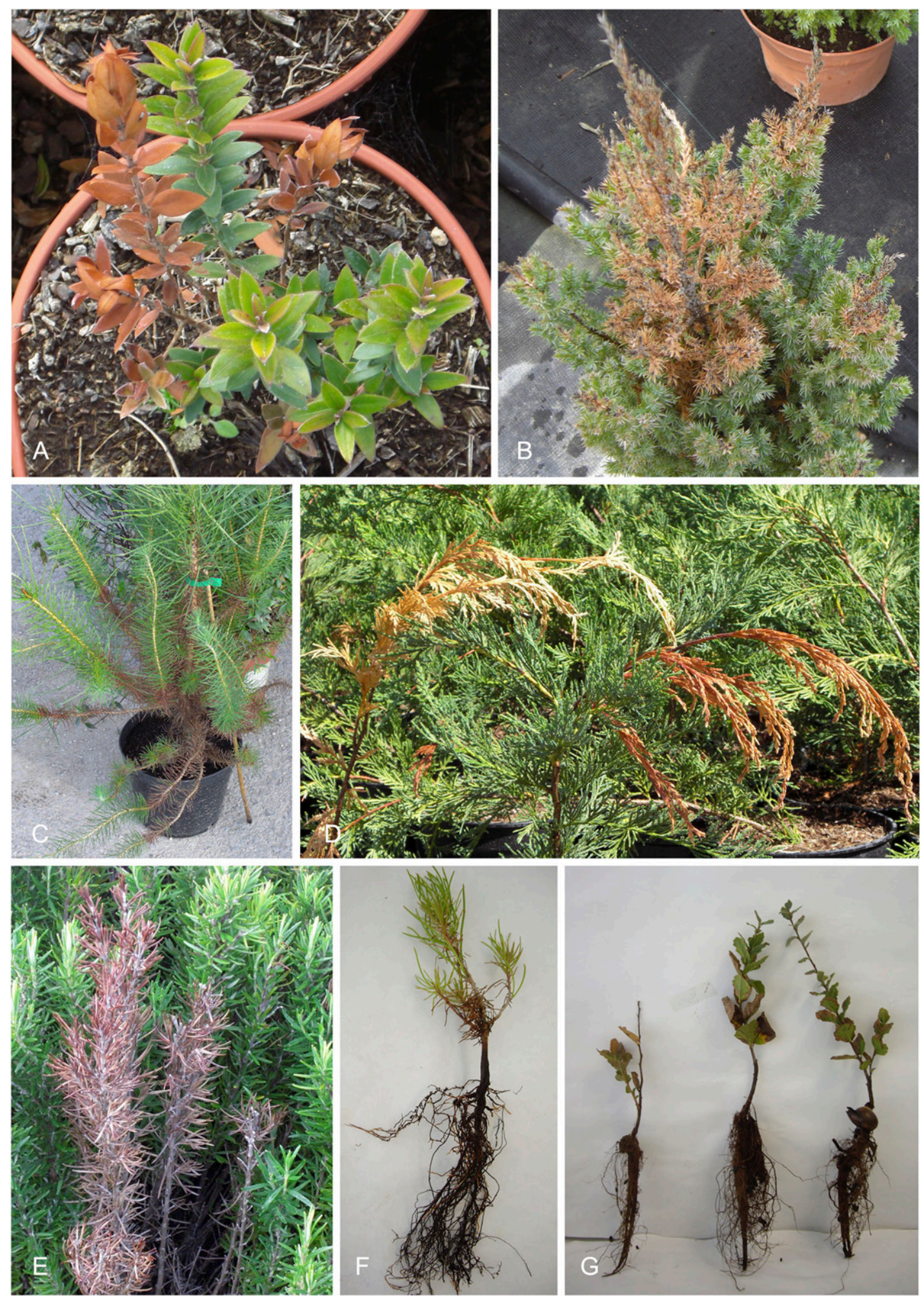

Fig. 1. Symptomatology of nursery plants from which Cylindrocarpon-like anamorphs were isolated. A, D, and E, shoot dieback on Myrtus sp. (A), Juniperus sp. (D), and Rosmarinus officinalis (E). B and C, Juniperus sp. and Pinus sp. dieback; apical dieback (B) and internal basal dieback (C). F, Pinus sp. seedlings showing reduced root system, including loss and rot of the feeder roots. G, Quercus ilex seedlings showing decline aerial symptoms, uneven growth, and a reduction of the root system. 
Table 1. Campylocarpon, Cylindrodendrum, Dactylonectria, and Ilyonectria isolates used in this study

\begin{tabular}{|c|c|c|c|c|c|c|c|c|}
\hline \multirow[b]{2}{*}{ Species } & \multirow[b]{2}{*}{ Strain number ${ }^{\mathbf{a}}$} & \multirow[b]{2}{*}{ Host } & \multirow{2}{*}{$\begin{array}{c}\text { Collected/isolated } \\
\text { by/year }\end{array}$} & \multirow[b]{2}{*}{ Location } & \multicolumn{4}{|c|}{ GenBank accession numbers } \\
\hline & & & & & ITS & $t u b 2$ & his3 & tefl \\
\hline $\begin{array}{l}\text { Campylocarpon } \\
\text { fasciculare }\end{array}$ & $\begin{array}{l}\text { CBS 112613; STE- } \\
\text { U 3970; C } 76\end{array}$ & Vitis vinifera & F. Halleen & $\begin{array}{l}\text { South Africa, Western } \\
\text { Cape, Riebeeck Kasteel }\end{array}$ & AY677301 & AY677221 & JF735502 & JF735691 \\
\hline $\begin{array}{l}\text { C. } \\
\quad \text { pseudofasciculare }\end{array}$ & $\begin{array}{l}\text { CBS 112679; } \\
\text { CPC5472; HJS- } \\
1227\end{array}$ & $V$. vinifera & F. Halleen & $\begin{array}{l}\text { South Africa, Western } \\
\text { Cape, Wellington }\end{array}$ & AY677306 & AY677214 & JF735503 & JF735692 \\
\hline \multirow[t]{2}{*}{$\begin{array}{l}\text { Cylindrodendrum } \\
\text { album }\end{array}$} & $\begin{array}{l}\text { CBS } 110655 \text {; VC- } \\
51\end{array}$ & Pine forest soil & $\begin{array}{l}\text { F. X. Prenafeta- } \\
\text { Boldú }\end{array}$ & $\begin{array}{l}\text { The Netherlands, } \\
\text { De Veluwe }\end{array}$ & KM231765 & KM232022 & KM231485 & KM231890 \\
\hline & $\begin{array}{l}\text { CBS 301.83; ATCC } \\
\text { 46842; } \\
\text { IMI 255534; } \\
\text { TRTC } \\
\text { 49165; UBC } 8265\end{array}$ & Fucus distichus & R. C. Summerbell & $\begin{array}{l}\text { Canada, British } \\
\text { Columbia, Vancouver, } \\
\text { Wreck Beach }\end{array}$ & KM231764 & KM532021 & KM231484 & KM231889 \\
\hline \multirow[t]{3}{*}{ C. alicantinum } & CBS 139518; Cyl-3 & Eriobotrya japonica & J. Armengol & $\begin{array}{l}\text { Spain, Alicante, Callosa } \\
\text { d'En Sarrià }\end{array}$ & KP456014 & KP400578 & KP639555 & KP452501 \\
\hline & Cyl-11 & E. japonica & J. Armengol & $\begin{array}{l}\text { Spain, Alicante, Callosa } \\
\text { d'En Sarrià }\end{array}$ & KP456017 & KP400581 & KP639558 & KP452504 \\
\hline & Cy-FO-25 & Quercus ilex & $\begin{array}{l}\text { P. Abad-Campos } \\
2011\end{array}$ & Spain, Valencia, Ayora & - & - & KX709593 & - \\
\hline \multirow[t]{2}{*}{ C. hubeiense } & $\begin{array}{l}\text { CBS 124071; } \\
\text { HMAS 98331, } \\
5620\end{array}$ & Rhododendron & $\begin{array}{l}\text { W. P. Wu, W. Y. } \\
\text { Zhuang \& Y. } \\
\text { Nong }\end{array}$ & China, Hubei & FJ560439 & FJ860056 & KR909093 & HM054090 \\
\hline & CBS 129.97 & Viscum album & W. Gams & $\begin{array}{l}\text { France, Dép. Jura, } \\
\text { Châtelneuf near } \\
\text { St. Laurent }\end{array}$ & KM231766 & KM232023 & KM231486 & KM231891 \\
\hline \multirow[t]{2}{*}{$\begin{array}{c}\text { Dactylonectria } \\
\text { alcacerensis }\end{array}$} & $\begin{array}{l}\text { CBS 129087; } \\
\text { Cy159 }\end{array}$ & $V$. vinifera & $\begin{array}{l}\text { A. Cabral \& } \\
\text { H. Oliveira }\end{array}$ & $\begin{array}{l}\text { Portugal, Alcácer de Sol } \\
\text { Torrão }\end{array}$ & JF735333 & AM419111 & JF735630 & JF735819 \\
\hline & Cy134; IAM Cy20-1 & $V$. vinifera & J. Armengol & $\begin{array}{l}\text { Spain, Ciudad Real, } \\
\text { Villarrubia de los Ojos }\end{array}$ & JF735332 & AM419104 & JF735629 & JF735818 \\
\hline D. anthuriicola & $\begin{array}{c}\text { CBS 564.95; PD } \\
\text { 95/1577 }\end{array}$ & Anthurium sp. & R. Pieters 1995 & Netherlands, Bleiswijk & JF735302 & JF735430 & JF735579 & JF735768 \\
\hline \multirow[t]{2}{*}{ D. estremocensis } & $\begin{array}{l}\text { CBS 129085; } \\
\text { Cy145 }\end{array}$ & $V$. vinifera & $\begin{array}{l}\text { C. Rego \& } \\
\text { T. Nascimento }\end{array}$ & Portugal, Estremoz & JF735320 & JF735448 & JF735617 & JF735806 \\
\hline & $\begin{array}{l}\text { CPC 13539; } \\
\text { 94-1685; } \\
\text { CCFC } 226730\end{array}$ & Picea glauca & R. C. Hamelin 1994 & Canada, Quebec & JF735330 & JF735458 & JF735627 & JF735816 \\
\hline \multirow[t]{2}{*}{ D. hispanica } & $\begin{array}{l}\text { CBS 142827; } \\
\text { Cy-FO-45 }\end{array}$ & Pinus halepensis & B. Mora-Sala 2011 & Spain, Valencia, Ayora & KY676882 & KY676876 & KY676864 & KY676870 \\
\hline & Су 228 & Ficus sp. & F. Caetano 2003 & Portugal, Lisbon & JF735301 & JF735429 & JF735578 & JF735767 \\
\hline D. hordeicola & CBS 162.89 & Hordeum vulgare & M. Barth & $\begin{array}{l}\text { Netherlands, } \\
\text { Noordoostpolder, } \\
\text { Marknesse, } \\
\text { Lovinhhoeve }\end{array}$ & AM419060 & AM419084 & JF735610 & JF735799 \\
\hline \multirow[t]{11}{*}{ D. macrodidyma } & $\begin{array}{l}\text { CBS 112615; STE- } \\
\text { U 3976; C98; } \\
\text { CPC 20709 }\end{array}$ & V. vinifera & F. Halleen & $\begin{array}{l}\text { South Africa, Western } \\
\text { Cape, Malmesbury, } \\
\text { Jakkalsfontein }\end{array}$ & AY677290 & AY677233 & JF735647 & JF735836 \\
\hline & $\begin{array}{l}\text { CBS 112601; STE- } \\
\text { U 3983; C } 82\end{array}$ & V. vinifera & F. Halleen 1999 & $\begin{array}{l}\text { South Africa, Western } \\
\text { Cape, Tulbagh }\end{array}$ & AY677284 & AY677229 & JF735644 & JF735833 \\
\hline & Cy-FO-1 & Q. faginea & $\begin{array}{l}\text { P. Abad-Campos } \\
2011\end{array}$ & Spain, Alicante, Alcoi & - & - & KX709497 & - \\
\hline & Cy-FO-9 & Q. ilex & $\begin{array}{l}\text { P. Abad-Campos } \\
2011\end{array}$ & Spain, Alicante, Alcoi & - & - & KX709498 & - \\
\hline & Cy-FO-10 & P. halepensis & $\begin{array}{l}\text { P. Abad-Campos } \\
2011\end{array}$ & Spain, Alicante, Alcoi & - & - & KX709499 & - \\
\hline & Cy-FO-13 & P. halepensis & $\begin{array}{l}\text { P. Abad-Campos } \\
2011\end{array}$ & $\begin{array}{l}\text { Spain, Valencia, Quart de } \\
\text { Poblet }\end{array}$ & - & - & KX709500 & - \\
\hline & Cy-FO-18 & Q. faginea & $\begin{array}{l}\text { P. Abad-Campos } \\
2011\end{array}$ & Spain, Valencia, Ayora & - & - & KX709501 & - \\
\hline & Cy-FO-19 & J. phoenicea & $\begin{array}{l}\text { P. Abad-Campos } \\
2011\end{array}$ & Spain, Valencia, Ayora & - & - & KX709502 & - \\
\hline & Cy-FO-20 & J. phoenicea & $\begin{array}{l}\text { P. Abad-Campos } \\
2011\end{array}$ & Spain, Valencia, Ayora & - & - & KX709503 & - \\
\hline & Cy-FO-23 & Q. ilex & $\begin{array}{l}\text { P. Abad-Campos } \\
2011\end{array}$ & Spain, Valencia, Ayora & - & - & KX709504 & - \\
\hline & $\mathrm{Cy}-\mathrm{FO}-24$ & Q. ilex & $\begin{array}{l}\text { P. Abad-Campos } \\
2011\end{array}$ & Spain, Valencia, Ayora & - & - & KX709505 & - \\
\hline
\end{tabular}

(Continued on next page)

\footnotetext{
${ }^{a}$ Ex-type culture indicated in bold. AR: Amy Y. Rossman personal collection; ATCC: American Type Culture Collection, USA; CBS: Westerdijk Fungal Biodiversity Institute, Utrecht, The Netherlands; CCFC: Canadian Collection of Fungal Cultures, Agriculture and Agri-Food Canada, Ottawa, Canada; CDC: Centers for Disease Control and Prevention, Atlanta, GA, USA; CPC: Culture collection of Pedro Crous, housed at CBS; Cy: Cylindrocarpon collection housed at Laboratório de Patologia Vegetal "Veríssimo de Almeida" - ISA, Lisbon, Portugal; DAOMC: Canadian Collection of Fungal Cultures, Canada; DiGeSA: Dipartimento di Gestione dei Sistemi Agroalimentari e Ambientali, Catania, Italy; DSM: Deutsche Sammlung von Mikroorganismen und Zellkulturen GmbH, Braunschweig, Germany; GJS: Gary J. Samuels collection; HJS: Hans-Josef Schroers collection; HMAS: Mycological Herbarium, Institute of Microbiology, Chinese Academy of Sciences; IAM: Instituto Agroforestal Mediterráneo, Universitat Politècnica de València, Spain; ICMP: International Collection of Microorganisms from Plants, Auckland, New Zealand; IMI: International Mycological Institute, CABI-Bioscience, Egham, Bakeham Lane, U.K.; JAT: J. A. Traquair collection; KIS: Agricultural Institute of Slovenia, Ljubljana, Slovenia; LYN: Lynchburg College, Biology Department, USA; MUCL: Mycothèque de l'Université Catholique de Louvain, Belgium; NBRC: NITE Biological Resource Center, Japan; NRRL: Agricultural Research Service Culture Collection, USA; NZ: Collection of L. Castlebury; PD: Collection of the Dutch National Plant Protection Organization (NPPO-NL), Wageningen, The Netherlands; STE-U: Stellenbosch University, South Africa; TRTC: Royal Ontario Museum Fungarium, Toronto, Ontario, Canada; UPSC: Fungal Culture Collection at the Botanical Museum, Uppsala University, Uppsala, Sweden; VKM: All-Russian Collection of Microorganisms, Russia.
} 
Table 1. (Continued from previous page)

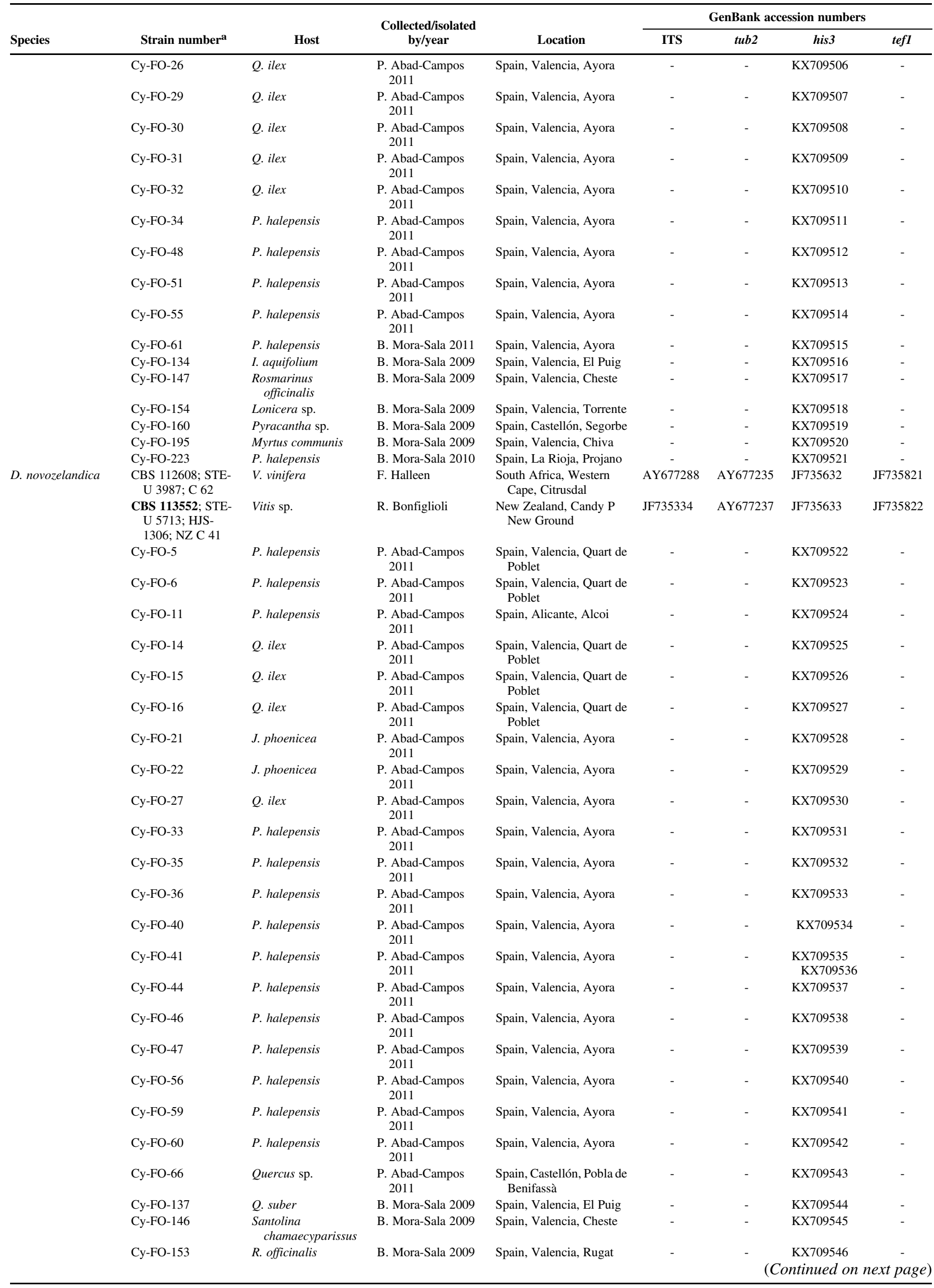


Table 1. (Continued from previous page)

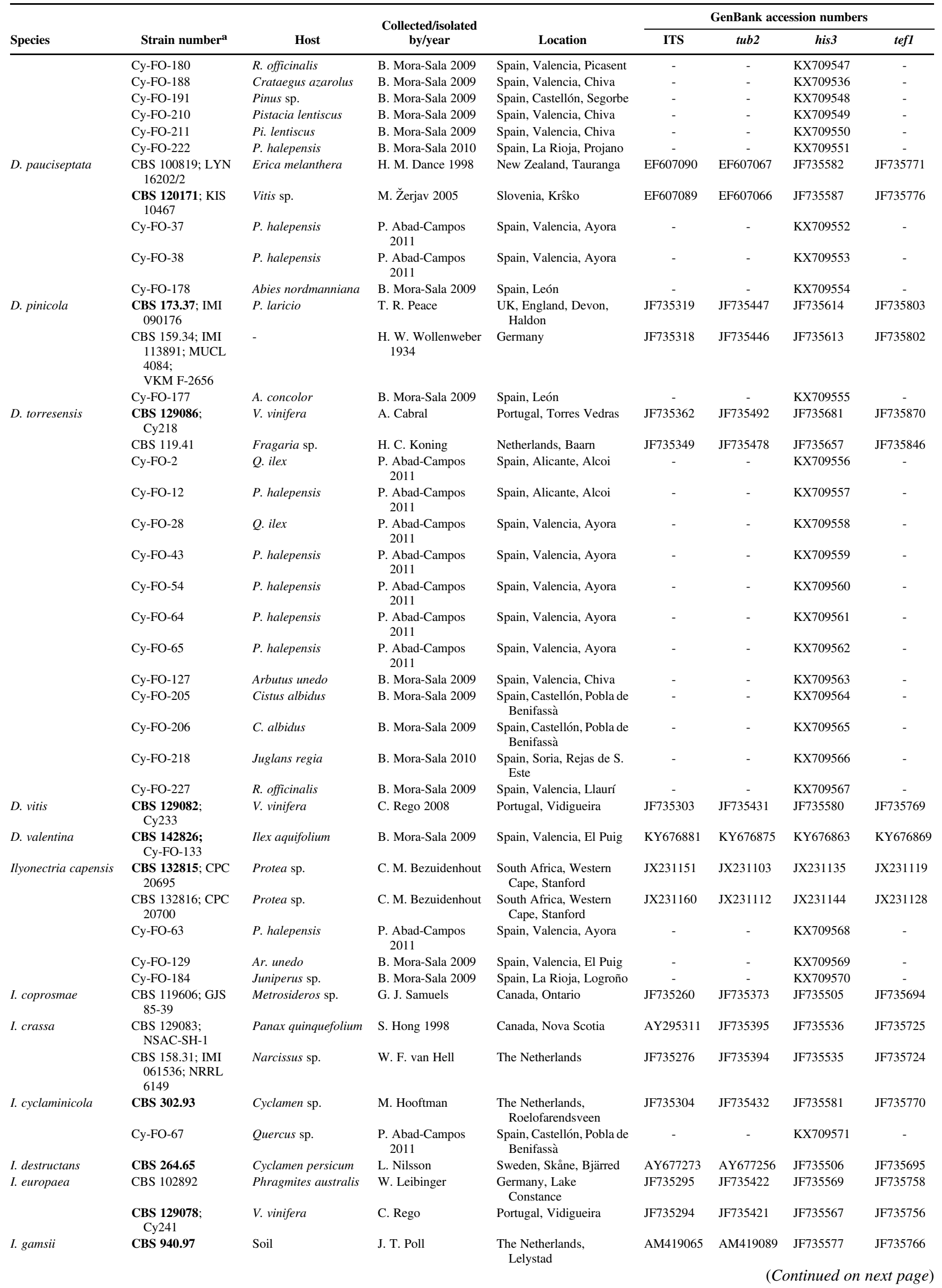


Table 1. (Continued from previous page)

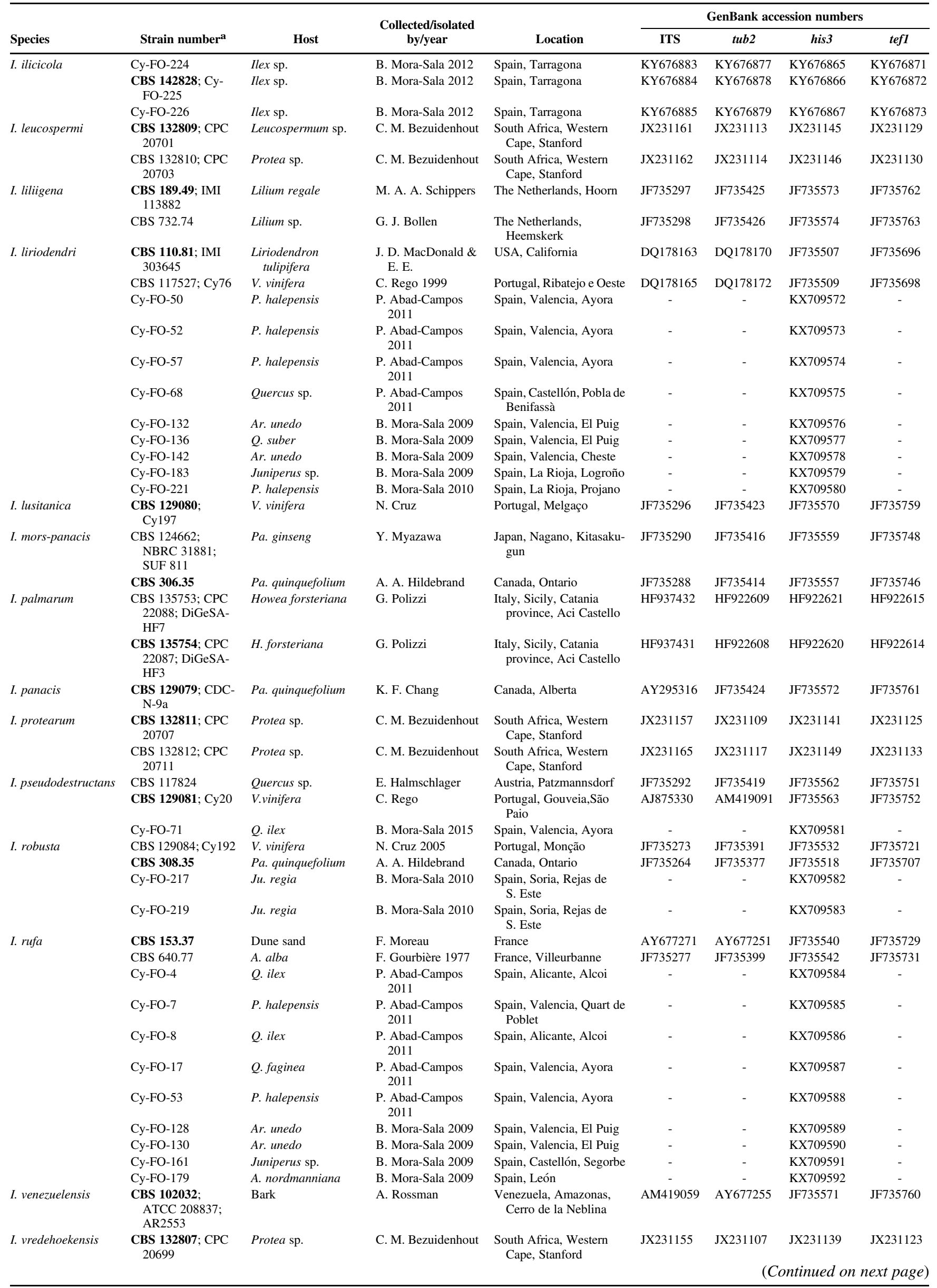


Table 1. (Continued from previous page)

\begin{tabular}{|c|c|c|c|c|c|c|c|c|}
\hline \multirow[b]{2}{*}{ Species } & \multirow[b]{2}{*}{ Strain number ${ }^{\mathbf{a}}$} & \multirow[b]{2}{*}{ Host } & \multirow{2}{*}{$\begin{array}{c}\text { Collected/isolated } \\
\text { by/year }\end{array}$} & \multirow[b]{2}{*}{ Location } & \multicolumn{4}{|c|}{ GenBank accession numbers } \\
\hline & & & & & ITS & tub2 & his3 & tefl \\
\hline & $\begin{array}{l}\text { CBS 132814; CPC } \\
20690^{\mathrm{b}}\end{array}$ & Protea sp. & C. M. Bezuidenhout & South Africa & JX231158 & JX231110 & JX231142 & JX231126 \\
\hline Neonectria candida & $\begin{array}{l}\text { CBS 182.36; IMI } \\
\text { 113893; UPSC } \\
1903\end{array}$ & Malus sylvestris & H. W. Wollenweber & - & JF735314 & JF735439 & JF735603 & JF735792 \\
\hline $\begin{array}{l}\text { N. candida, } \\
\text { authentic strain of } \\
\text { C. obtusiusculum } \\
\text { (=C. magnusianum) }\end{array}$ & $\begin{array}{l}\text { CBS 151.29; IMI } \\
\text { 113894; } \\
\text { MUCL 28083; } \\
\text { MUCL 28094 }\end{array}$ & Ma. sylvestris & H. W. Wollenweber & UK, England, Cambridge & JF735313 & JF735438 & JF735602 & JF735791 \\
\hline $\begin{array}{l}N . \text { ditissima, } \\
\text { authentic strain } \\
\text { of } C \text {. willkommii }\end{array}$ & $\begin{array}{l}\text { CBS 226.31; IMI } \\
\quad 113922\end{array}$ & Fagus sylvatica & H. W. Wollenweber & Germany, Tharandt & JF735309 & DQ789869 & JF735594 & JF735783 \\
\hline $\begin{array}{l}N \text {. ditissima, } \\
\text { representative } \\
\text { strain of } N . \\
\text { galligena }\end{array}$ & CBS 835.97 & Salix cinerea & W. Gams 1997 & $\begin{array}{l}\text { Belgium, Marais de } \\
\text { Sampant }\end{array}$ & JF735310 & DQ789880 & JF735595 & JF735784 \\
\hline$N$. major , type strain & $\begin{array}{l}\text { CBS 240.29; IMI } \\
113909\end{array}$ & Alnus incana & H. W. Wollenweber & Norway & JF735308 & DQ789872 & JF735593 & JF735782 \\
\hline \multirow{3}{*}{$\begin{array}{l}N \text {. neomacrospora } \\
\text { representative } \\
\text { strain }\end{array}$} & $\begin{array}{l}\text { CBS 324.61; DSM } \\
\text { 62489; IMB } 9628\end{array}$ & A. concolor & J. A. von Arx & Netherlands, Zwolle & JF735312 & DQ789875 & JF735599 & JF735788 \\
\hline & CBS 503.67 & A. alba, wood & F. Roll-Hansen & Norway, Hordaland, Fana & AY677261 & JF735436 & JF735600 & JF735789 \\
\hline & $\begin{array}{l}\text { CBS 118984; GJS } \\
\text { 03-28 }\end{array}$ & $\begin{array}{l}\text { Arceuthobium } \\
\text { tsugense }\end{array}$ & L. Reitman 2005 & $\begin{array}{l}\text { Canada, British } \\
\text { Columbia, Vancouver } \\
\text { Island, Spider Lake }\end{array}$ & JF735311 & DQ789882 & JF735598 & JF735787 \\
\hline \multirow[t]{2}{*}{ N. obtusispora } & $\begin{array}{l}\text { CBS 183.36; IMI } \\
\quad 113895\end{array}$ & Solanum tuberosum & $\begin{array}{l}\text { H. W. Wollenweber } \\
1936\end{array}$ & Germany & AM419061 & AM419085 & JF735607 & JF735796 \\
\hline & $\begin{array}{l}\text { CPC 13544; DAOM } \\
182772 ; \text { JAT } \\
1366\end{array}$ & Prunus armenica & J. A. Traquair 1982 & Canada, Ontario, Ruthven & AY295306 & JF735443 & JF735608 & JF735797 \\
\hline \multirow[t]{4}{*}{ N. quercicola } & $\begin{array}{l}\text { CBS 143704; } \\
\text { Cy-FO-3 }\end{array}$ & Q. ilex & $\begin{array}{l}\text { P. Abad-Campos } \\
2011\end{array}$ & Spain, Alicante, Alcoi & KY676880 & KY676874 & KY676862 & KY676868 \\
\hline & $\begin{array}{l}\text { CPC 13530; DAOM } \\
\text { 185722; JAT } \\
1591\end{array}$ & Pyrus sp. & J. A. Traquair 1983 & Canada, Ontario, Harrow & AY295302 & JF735441 & JF735605 & JF735794 \\
\hline & $\begin{array}{l}\text { CPC 13531; CCFC } \\
\text { 226722; DAOM } \\
\text { 226722; CR6 }\end{array}$ & $\begin{array}{l}\text { Pseudotsuga } \\
\text { menziesii }\end{array}$ & P. Axelrood & Canada, British Columbia & AY295301 & JF735442 & JF735606 & JF735795 \\
\hline & CR21 & Ps. menziesii & P. Axelrood & Canada, British Columbia & JF735315 & JF735440 & JF735604 & JF735793 \\
\hline Neonectria sp.1 & $\begin{array}{l}\text { CPC 13545; DAOM } \\
\text { 185212; \# } 5\end{array}$ & Pyrus sp. & $\begin{array}{l}\text { J. A. Traquair \& } \\
\text { B. Harrison } 1982\end{array}$ & Canada, Ontario, Harrow & AY295303 & JF735437 & JF735601 & JF735790 \\
\hline
\end{tabular}

both directions by the Macrogen Inc. Sequencing Center (The Netherlands). Sequences were assembled and edited to resolve ambiguities and consensus sequences for all isolates were compiled into a single file (Fasta format) using Sequencher software v. 5.3 (Gene Codes Corporation, Ann Arbor, MI, U.S.A.).

Phylogenetic analysis was first conducted on the his 3 singlelocus alignment for all isolates obtained in this study, as this locus was reported to be very informative (Cabral et al. 2012a). For cases where was not possible to infer species for a specific isolate with only the his 3 single-locus phylogeny, a combined alignment of the four loci (his3, ITS, tub2, and tefl) was also analyzed. GenBank sequences (Table 1) from different species of Cylindrodendrum, Dactylonectria, Ilyonectria, and Neonectria were selected based on their high similarity with our query sequences using MegaBLAST. These were added to the sequences obtained and aligned using MAFFT version 7.305 implemented on CIPRES Science Gateway V 3.3 (Miller et al. 2010) and edited manually, if necessary, using MEGA 7.0.26 (Kumar et al. 2016). The alignments for each locus were combined in a single file using the program SequenceMatrix 1.8 (Vaidya et al. 2011). The best nucleotide substitution model settings for each locus were determined by jModelTest 2.1.10 (Darriba et al. 2012), with the following likelihood settings: number of substitution schemes $=3$ (24 models), base frequencies $(+\mathrm{F})$, proportion of invariable sites $(+\mathrm{I})$, and rate variation among sites $(+\mathrm{G})(\mathrm{nCat}=4)$, using the Akaike information criterion (AIC). The Bayesian analyses of the combined four-loci dataset and individual locus data were performed with MrBayes v. 3.2.1 (Ronquist et al. 2012) based on the results of the jModelTest. The Markov Chain Monte Carlo sampling (MCMC) analysis of four chains started in parallel from a random tree topology (Rodriguez et al. 1990). The number of generations was set at $10 \mathrm{M}$ and the run was stopped automatically when the average standard deviation of split frequencies fell below 0.01 . Trees were saved each 1,000 generations. Burn-in was set at $25 \%$, after which the likelihood values were stationary and the remaining trees were used to calculate posterior probabilities. Trees from different runs were then combined and summarized in a majority rule $50 \%$ consensus tree. Maximum likelihood (ML) was implemented in the CIPRES Science Gateway V 3.3 (Miller et al. 2010) using RAxML-HPC v.8 on XSEDE (8.2.9) using the GTRCAT model and 1,000 rapid bootstrap inferences were done.

Both analyses were performed, rooting the trees to Campylocarpon (Ca.) fasciculare (CBS 112613) and Ca. pseudofasciculare (CBS 112679) and tree topologies were compared on http://phylo. io (Robinson et al. 2016).

Sequences derived in this study were lodged in GenBank, the alignments and phylogenetic trees in TreeBASE under study number S22022 (https://treebase.org/), and taxonomic novelties in MycoBank (www.MycoBank.org) (Crous et al. 2004a). GenBank accession numbers of the isolates collected during this study are listed in Table 1.

\section{Results}

Phylogenetic analysis. One hundred and three isolates were amplified with the primers CYLH3F and CYLH3R and approximately $500 \mathrm{bp}$ were obtained for all. The his 3 single-locus alignment contains 427 aligned characters (including gaps), from which 195 characters were parsimony-informative, 17 were variable but parsimony-uninformative, 
and 212 were constant. The AIC best-fit nucleotide substitution model identify by jModelTest was general time reversible model with inverse gamma rates $(\mathrm{GTR}+\mathrm{I}+\mathrm{G})$. The Bayesian consensus tree and ML tree had similar topology, and therefore only the Bayesian consensus tree is presented with bootstrap support values (BS) and posterior probability values (PP). The phylogenetic analysis contained a total of 174 ingroup taxa and two outgroup taxa (Ca. fasciculare [CBS112613] and Ca. pseudofasciculare [CBS112679]).

The phylogeny obtained with his 3 alignment resulted in four major clades: the first major clade comprised the isolates from the genus Ilyonectria; the second, isolates from the genus Neonectria; the third,

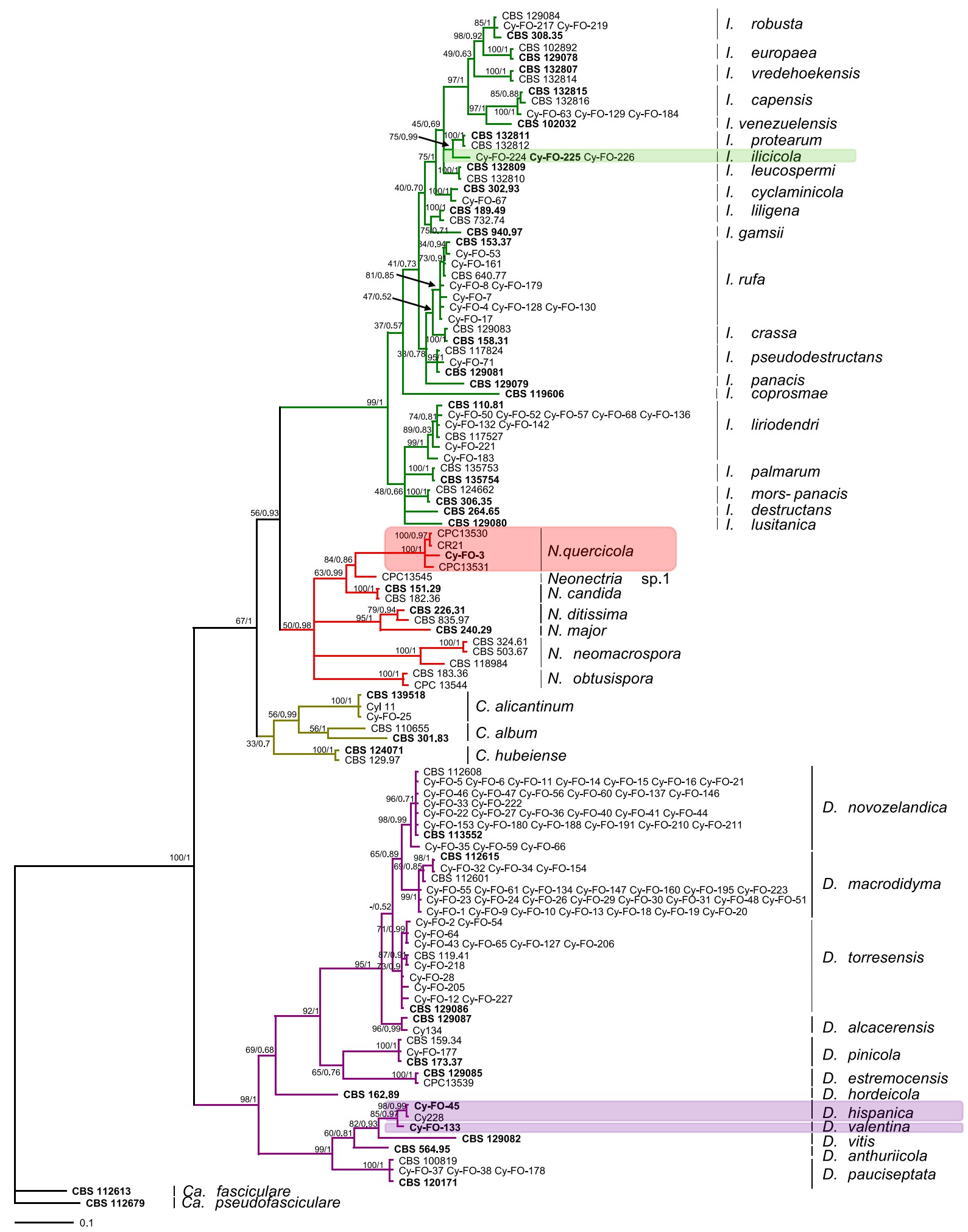

Fig. 2. Fifty percent majority rule consensus tree from a Bayesian analysis based on the alignment of partial histone $\mathrm{H} 3$ gene sequences from the 103 Cylindrocarpon-like asexual morphs obtained from forest nurseries, and additional sequences of Cylindrodendrum, Dactylonectria, llyonectria, and Neonectria species. The RAxML bootstrap support and Bayesian posterior probability values are indicated at the nodes (ML/PP). The tree was rooted to Campylocarpon fasciculare (CBS 112613) and C. pseudofasciculare (CBS 112679). The scale bar indicates 0.1 expected changes per site. Ex-type cultures are indicated in bold. Colors are used to indicate clades from the same genera Ca. - Campylocarpon, C. - Cylindrodendrum, D. - Dactylonectria, I. - Ilyonectria, N. - Neonectria. Tentative new species are indicated in shaded boxes. 
isolates from the genus Cylindrodendrum; and the fourth, isolates from the genus Dactylonectria (Fig. 2). About $71 \%$ of the isolates obtained in this study belonged to the genus Dactylonectria and included D. hispanica (0.97\%), D. macrodidyma $(24.27 \%)$,
D. novozelandica $(29.13 \%)$, D. pauciseptata $(2.91 \%)$, D. pinicola $(0.97 \%), D$. torresensis $(11.65 \%)$, and D. valentina $(0.97 \%)$. The genus Ilyonectria included $27.18 \%$ of the isolates I. capensis $(2.91 \%)$, I. cyclaminicola $(0.97 \%)$, I. ilicicola $(2.91 \%)$, I. liriodendri $(8.74 \%)$,

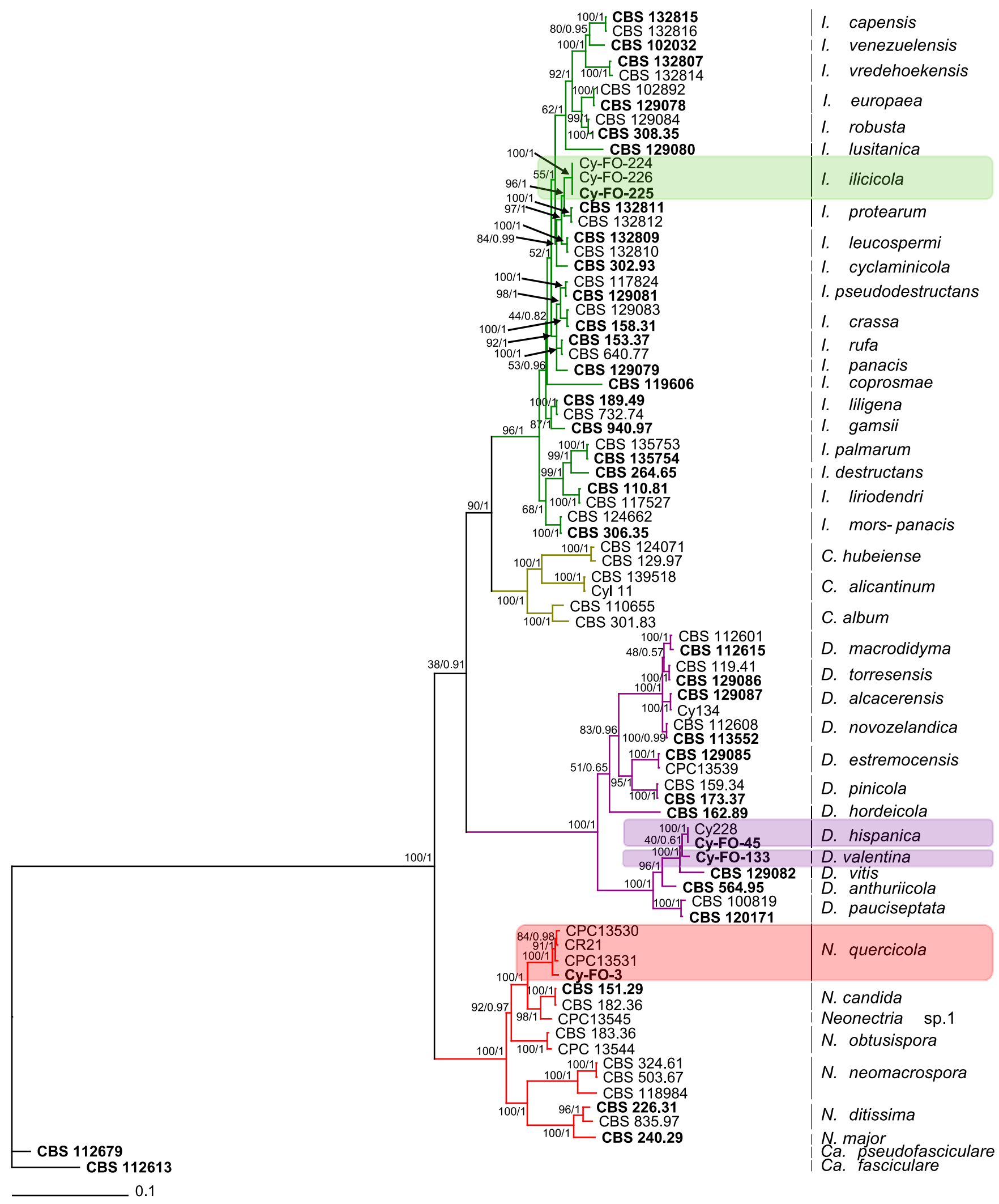

Fig. 3. Fifty percent majority rule consensus tree from a Bayesian analysis based on the combined four gene dataset (ITS, tub2, his3, and tef1). The RAxML bootstrap support and Bayesian posterior probability values are indicated at the nodes (ML/PP). The tree was rooted to Campylocarpon fasciculare (CBS 112613) and C. pseudofasciculare (CBS 112679). The scale bar indicates 0.1 expected changes per site. Ex-type cultures are indicated in bold. Colors are used to indicate clades from the same genera Ca. - Campylocarpon, C. - Cylindrodendrum, D. - Dactylonectria, I. - Ilyonectria, N. - Neonectria. New species are indicated in shaded boxes. 
I. pseudodestructans (0.97\%), I. robusta (1.94\%), and I. rufa (8.74\%). The genus Neonectria contained one species, N. quercicola $(0.97 \%)$. Cylindrodendrum alicantinum (C. alicantinum) (0.97\%) was the only species of the genus Cylindrodendrum.

Six isolates (Cy-FO-3, Cy-FO-45, Cy-FO-133, Cy-FO-224, CyFO-225, and Cy-FO-226) could not be identified to the species level employing the his3 sequences (Fig. 2). Therefore, the ITS, tub2, and tefl regions were analyzed additionally, and these sequences were concatenated with those obtained from the his 3 region for their identification (Fig. 3). The four loci alignment contained 79 taxa (including the two outgroups) and 1,935 aligned characters (including gaps), from which 718 characters were parsimony-informative, 83 were variable but parsimony-uninformative, and 1,101 were constant. The AIC best-fit nucleotide substitution model identified by jModelTest was GTR+I+G model for ITS and his 3 and GTR+G for $t u b 2$ and tef1.

The Bayesian and ML consensus trees obtained with the four-loci alignment confirmed the existence of four novel taxa within our set of isolates.

Taxonomy. Based on the phylogenetic analysis and morphological characters, two new species of Dactylonectria, one species of Ilyonectria, and one species of Neonectria are described (Figs. 2, 3 , and 4). No perithecia were observed in the homothallic or heterothallic crosses performed.

D. hispanica B. Mora-Sala, A. Cabral, J. Armengol \& P. AbadCampos, sp. nov. MycoBank MB822023 (Fig. 5). Etymology: Name refers to Spain, where the fungus was isolated.

Diagnosis: Morphologically D. hispanica, can be distinguished by its slightly larger 3-septate macroconidia when compared with
D. vitis and $D$. valentina. Fourteen polymorphisms can distinguish $D$. hispanica from $D$. valentina: five in tub2 locus at position 27 (A:T), 136 (G:A), 206 (A:G), 335 (T:C), and 434 (C:A), five in his3 locus at position 94 (C:T), 104 (T:C); 214 (T:C); 291 (T:C), and 395 (C:T); and four in tefl locus at position 224 (G:A), 266 (A:T), 289 (T:A), and 291 (A:C).

Typus: Spain: Valencia, Ayora, on Pinus halepensis (complete roots), 2011, B. Mora-Sala (CBS H-23154 - holotype; CBS $142827=$ Cy-FO-45 - ex-type culture).

Conidiophores simple. Complex conidiophores not observed. Simple conidiophores arising laterally or terminally from aerial mycelium, solitary to aggregated, unbranched or sparsely branched with up to four phialides, 1 - to 2 -septate, 40 to $75 \mu \mathrm{m}$ long; phialides monophialidic, cylindrical, tapering toward the apex, 16 to $28 \mu \mathrm{m}$ long, 2.0 to $3.0 \mu \mathrm{m}$ wide at the base, 3.0 to $4.0 \mu \mathrm{m}$ at the widest point, 1.5 to $2.5 \mu \mathrm{m}$ near the aperture.

Macroconidia (1 to) 3-septate, straight or minutely curved, cylindrical with both ends more or less broadly rounded, mostly with a visible centrally located to laterally displaced hilum; 1-septate (26-) 31 to $37(-53) \times(7.0-) 7.5$ to $8.5(-9.0) \mu \mathrm{m}($ av. $34 \times 8 \mu \mathrm{m}), \mathrm{L} / \mathrm{W}$ ratio (3-) 3.9 to 4.7 (-6.5) (av. 4.3), 2-septate (30.5-) 37 to $43(-53) \times(6-)$ 7.5 to $8.5(-9) \mu \mathrm{m}$ (av. $40 \times 8 \mu \mathrm{m}$ ), L/W ratio (3.5-) 4.5 to $5.5(-7)$ (av. 5.0), and 3-septate macroconidia (39-) 45 to $47(-58) \times(6.5-)$ 7.8 to $8.2(-9.5) \mu \mathrm{m}$ (av. $46 \times 8 \mu \mathrm{m})$, L/W ratio (4.5-) 5.5 to 6 $(-7.5)$ (av. 5.8). Macroconidia formed in heads or as flat domes of slimy masses.

Microconidia rarely formed, aseptate to 1-septate with a minutely or clearly laterally displaced hilum; aseptate microconidia ellipsoidal
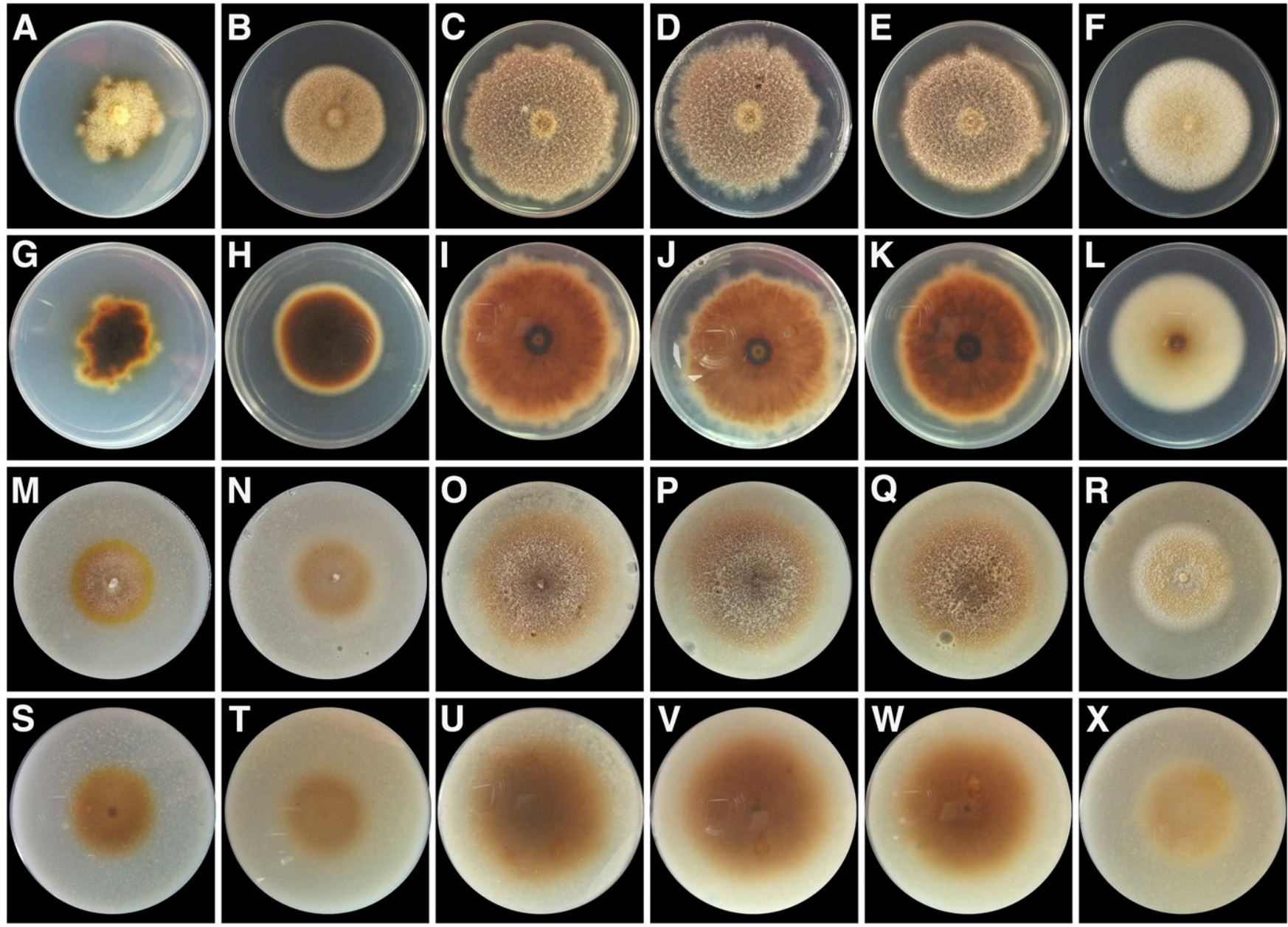

Fig. 4. Ten-day-old colonies grown at $20^{\circ} \mathrm{C}$ in darkness on PDA (A-F upper face; G-L bottom face) and oatmeal agar (M-R upper face; S-Y bottom face) of: Dactylonectria hispanica isolate Cy-FO-45 (A, G, M, and S); D. valentina isolate Cy-FO-133 (B, H, N, and T); llyonectria ilicicola isolates Cy-FO-224 (C, I, O, and U), Cy-FO-225 (D, J, P, and V), and Cy-FO226 (E, K, Q, and $\mathbf{W})$, and Neonectria quercicola Cy-FO-3 (F, L, R, and $\mathbf{X})$. 
to fusiform (9.5-) 10.5 to $14(-17) \times(5.5-) 6$ to 7 (-7.5) (av. $12.2 \times$ $6.5 \mu \mathrm{m})$, L/W ratio (1.5-) 1.7 to 2.1 (-2.2) (av. 1.8); 1-septate, fusiform to subcylindrical (14-) 19 to $21.5(-24.5) \times(6-) 7$ to $7.5(-8.5)$ (av. $20.2 \times 7.2 \mu \mathrm{m}$ ), $\mathrm{L} / \mathrm{W}$ ratio (2-) 2.6 to 3.0 (-3.5) (av. 2.8). Chlamydospores observed on SNA; globose to subglobose to ellipsoidal, 7 to $10 \times 6$ to $9 \mu \mathrm{m}$ diameter, smooth but often appearing rough due to deposits, thick-walled, in chains or in clumps, hyaline, becoming slightly brown.

Culture characteristics: Mycelium felty with low to average density (OA) or average to strong density (PDA). Surface on OA buff to sepia with sparse cinnamon aerial mycelium; margin luteous. Surface on PDA honey to buff; margin buff. Zonation absent, transparency homogeneous and margins even (OA) and uneven (PDA). Reverse similar to surface, except in color, sepia to cinnamon (PDA). Colonies on PDA grow poorly, less than $1 \mathrm{~mm}$ diameter at $5^{\circ} \mathrm{C}$ after 7 days. Optimum temperature at $25^{\circ} \mathrm{C}$, when colonies reach $28 \mathrm{~mm}$ diameter, after 7 days. Colony diameter was $9 \mathrm{~mm}$ at $30^{\circ} \mathrm{C}$ after 7 days. No growth was observed at $35^{\circ} \mathrm{C}$.

Host and distribution: Pinus halepensis (roots) (Spain, Valencia).

Notes: $D$. hispanica is closely related to $D$. valentina and $D$. vitis based on phylogenetic inference. The morphology of these species is very similar, but $D$. hispanica can be distinguished by its slightly larger 3-septate macroconidia when compared with $D$. vitis (34.9-) 41.6 to $43.5(-51.6) \times(6.2-) 7.9$ to $8.2(-9.5) \mu \mathrm{m}(\mathrm{av} .=42.5 \times$ $8.0 \mu \mathrm{m}$; Cabral et al. 2012a) and D. valentina (30-) 35.5 to 37 $(-44) \times(6-) 7.5$ to $8(-9.0) \mu \mathrm{m}$ (av. $36.3 \times 7.6 \mu \mathrm{m}$; this study). No complex conidiophores or penicillate conidiophores with aseptate microconidia were observed in D. hispanica. The isolate Cy228 is classified as D. hispanica, as it forms a clade very well supported $(\mathrm{BS}=100 \%$ and $\mathrm{PP}=1.0)$ with the isolate $\mathrm{Cy}-\mathrm{FO}-45$.

D. valentina B. Mora-Sala, A. Cabral, J. Armengol \& P. AbadCampos, sp. nov. MycoBank MB822024 (Fig. 6). Etymology: Name refers to the province of Valencia, Spain, where the fungus was isolated.

Diagnosis: The morphologically $D$. valentina can be distinguished by its slightly smaller 3 -septate macroconidia when compare with $D$. vitis and $D$. hispanica, and for the absence of luteous margin in
OA plates. Fourteen nucleotide differences can distinguish $D$. valentina from $D$. hispanica (as described in diagnosis of $D$. hispanica).

Typus: Spain: Valencia, El Puig, 2009, on Ilex aquifolium (complete roots), B. Mora-Sala (CBS H-23155 - holotype; CBS $142826=$ Cy-FO-133 - ex-type culture).

Conidiophores simple or complex. Simple conidiophores arising laterally or terminally from aerial mycelium, solitary to aggregated, unbranched or sparsely branched with up to four phialides, 1- to 4septate, 55 to $130 \mu \mathrm{m}$ long; phialides monophialidic, cylindrical, tapering toward the apex, 15 to $30.5 \mu \mathrm{m}$ long, 2.1 to $3.2 \mu \mathrm{m}$ wide at the base, 3.1 to $4.5 \mu \mathrm{m}$ at the widest point, 1.5 to $3 \mu \mathrm{m}$ near the aperture. Conidiophores forming aseptate microconidia arising from mycelium on agar surface, 1- to 4-septate, with a terminal arrangement of phialides, ranging from 2 to a dense cluster; sparsely branched or penicillate; monophialides narrowly flask-shaped, typically with widest point near the middle, 9 to $17 \mu \mathrm{m}$ long, 1.5 to $3.0 \mu \mathrm{m}$ wide at the base, 2.0 to $3.5 \mu \mathrm{m}$ at widest point, 1.0 to $2.0 \mu \mathrm{m}$ near the apex. Sporodochial conidiophores irregularly branched; phialides more or less cylindrical but slightly tapering toward the tip, or narrowly flaskshaped, with widest point near the middle, 14 to $20 \mu \mathrm{m}$ long, 2.5 to $3.5 \mu \mathrm{m}$ wide at the base, 3.0 to $4.5 \mu \mathrm{m}$ at widest point, 1.5 to $2.5 \mu \mathrm{m}$ near the apex. Macroconidia (1 to) 3-septate, straight or minutely curved, cylindrical with both ends more or less broadly rounded, mostly with a visible centrally located to laterally displaced hilum; 1-septate $(18-) 25$ to $28(-33.5) \times(5.5-) 6.5$ to $7(-8.5) \mu \mathrm{m}$ (av. $26.3 \times 6.9 \mu \mathrm{m}), \mathrm{L} / \mathrm{W}$ ratio (3-) 3.7 to 4.2 (-5) (av. 3.9), 2-septate (26-) 30 to $32(-35.5) \times(6.5-) 7.5$ to $8(-9) \mu \mathrm{m}$ (av. $31.1 \times$ $7.6 \mu \mathrm{m}$ ), L/W ratio (3.0-) 3.9 to 4.3 (-5) (av. 4.1), and 3-septate macroconidia (30-) 35.5 to $37(-44) \times(6-) 7.5$ to $8(-9.0) \mu \mathrm{m}$ (av. $36.3 \times$ $7.6 \mu \mathrm{m}$ ), L/W ratio (3.5-) 4.5 to 5 (-6.5) (av. 4.8). Macroconidia formed in heads or as flat domes of slimy masses. Microconidia aseptate to 1-septate with a minutely or clearly laterally displaced hilum; aseptate microconidia subglobose to oval (3.5-) 5 to $5.5(-7.5) \times(3-)$ 3.9 to $4.1(-4.5) \mu \mathrm{m}$ (av. $5.2 \times 4 \mu \mathrm{m}), \mathrm{L} / \mathrm{W}$ ratio (1-) 1.2 to $1.4(-2)$ (av. 1.3); 1-septate microconidia, rarely formed, fusiform to subcylindrical (14-) 15 to $17(-18.5) \times(4.5-) 4.7$ to $5.5(-6) \mu \mathrm{m}(\mathrm{av}$. $16 \times 5 \mu \mathrm{m}), \mathrm{L} / \mathrm{W}$ ratio $(2.5-) 2.8$ to $3.3(-3.5)$ (av. 3.0).
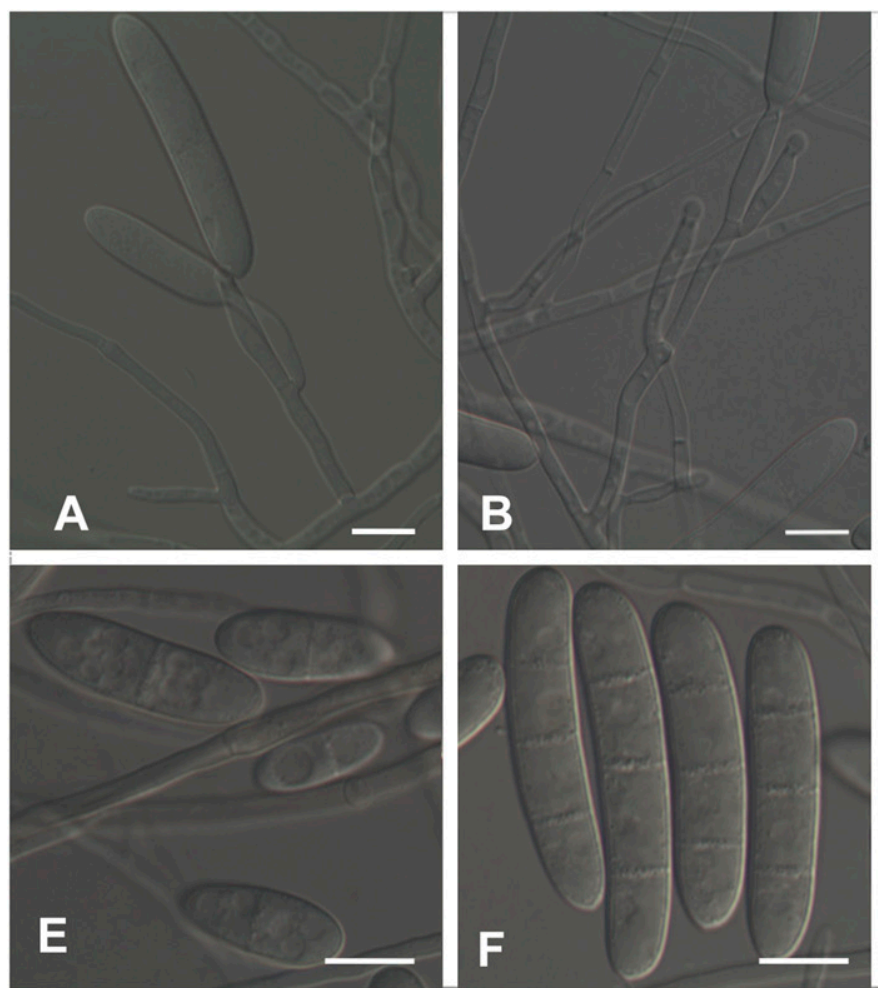
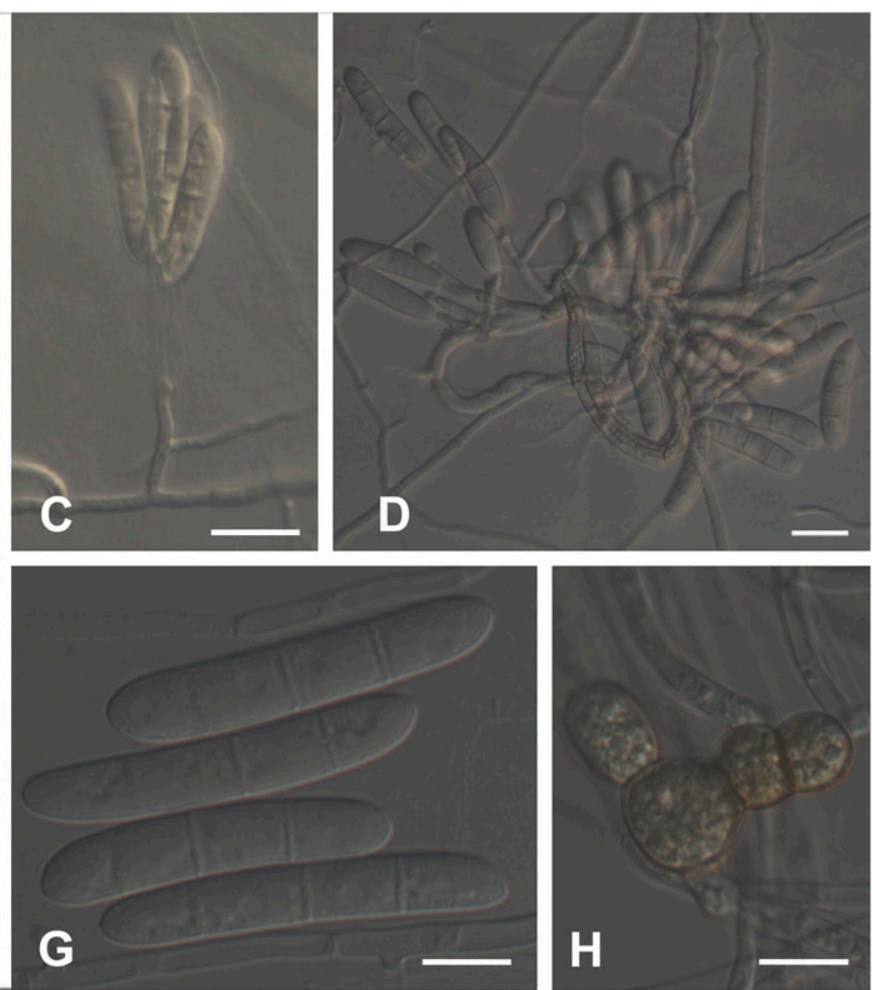

Fig. 5. Dactylonectria hispanica (ex-type culture Cy-FO-45). A-D, Simple, sparsely branched conidiophores of the aerial mycelium. E-G, Micro- and macroconidia. H, Chlamydospores in mycelium. Scale bars: $C, D=20 \mu \mathrm{m} ; \mathrm{A}-\mathrm{B}, \mathrm{E}-\mathrm{H}=10 \mu \mathrm{m}$. 
Chlamydospores observed in the bottom of SNA plate; globose to subglobose to ellipsoidal, 9 to $19 \times 7$ to $12 \mu \mathrm{m}$ diameter, smooth but often appearing rough due to deposits, thick-walled, mainly in chains or in clumps, hyaline, becoming slightly brown in the outer wall.

Culture characteristics: Mycelium felty with density low to average (OA) and average to strong (PDA). Surface on OA sienna, with sparse, saffron aerial mycelium, and buff growth at margin. Surface on PDA chestnut, with sienna aerial mycelium, with buff margin. Zonation was absent, transparency was homogeneous, and growth margin even. Reverse similar to surface, except in color, chestnut to sienna on PDA. Colonies on PDA grow poorly, $1 \mathrm{~mm}$ diameter at $5^{\circ} \mathrm{C}$ after 7 days. Optimum temperature at $25^{\circ} \mathrm{C}$, when colonies reach $35 \mathrm{~mm}$ diameter after 7 days. Colony diameter was $8 \mathrm{~mm}$ at $30^{\circ} \mathrm{C}$ after 7 days. No growth was observed at $35^{\circ} \mathrm{C}$.

Host and distribution: Ilex aquifolium (roots) (Spain, Valencia).

Ilyonectria ilicicola B. Mora-Sala, A. Cabral, J. Armengol \& P. Abad-Campos, sp. nov. MycoBank MB822025 (Fig. 7). Etymology: Name refers to the plant host genus, Ilex, from which this fungus was isolated.

Diagnosis: Ilyonectria ilicicola can be distinguish morphologically from I. cyclaminicola, I. leucospermi, and I. protearum by having slightly larger and narrower macroconidia. This taxa is best distinguish by tub2 and his3 genes.
Typus: Spain: Tarragona, 2012, on Ilex sp. roots, B. Mora-Sala (CBS H-23156 - holotype; CBS 142828 = Cy-FO-225 - ex-type culture)

Conidiophores simple or complex. Simple conidiophores arising laterally or terminally from aerial mycelium, solitary to aggregated, unbranched or sparsely branched with up to three phialides, 1- to 3-septate, 49 to $178 \mu \mathrm{m}$ long; phialides monophialidic, cylindrical, tapering toward the apex, 26 to $66 \mu \mathrm{m}$ long, 2 to $4 \mu \mathrm{m}$ wide at the base, 2.5 to $4.5 \mu \mathrm{m}$ at the widest point, 1.5 to $3 \mu \mathrm{m}$ near the aperture.

Complex conidiophores aggregated in sporodochia. Sporodochia consist of a pulvinate mass of short conidiophores, irregularly branched; phialides cylindrical, tapering toward the apex, 12 to $30 \mu \mathrm{m}$ long, 1.5 to $2.5 \mu \mathrm{m}$ wide at the base, 2.0 to $2.5 \mu \mathrm{m}$ at the widest point, and 1 to $2 \mu \mathrm{m}$ wide at the apex.

Macroconidia 1 (to 3)-septate, straight, cylindrical, with both ends obtusely rounded, base sometimes with a visible, centrally located to laterally displaced hilum; 1-septate macroconidia (19.5-) 25 to 26 $(-32.5) \times(3.5-) 5$ to $5.5(-6.5) \mu \mathrm{m}$ (av. $25.5 \times 5.2 \mu \mathrm{m}), \mathrm{L} / \mathrm{W}$ ratio (3.5-) 4.9 to $5.1(-7)$ (av. 5.0); 2-septate macroconidia (26.5-) 30 to $31.5(-35.5) \times(5-) 5.5$ to $6(-7) \mu \mathrm{m}($ av. $30.7 \times 5.7 \mu \mathrm{m}), \mathrm{L} / \mathrm{W}$ ratio (4.5-) 5.2 to 5.7 (-7) (av. 5.4); and 3-septate macroconidia (28-) 31.5 to $34(-40.5) \times(4.5-) 5.7$ to $6.2(-7) \mu \mathrm{m}($ av. $32.9 \times 5.9 \mu \mathrm{m})$, L/W ratio (4.5-) 5.3 to $5.9(-7.75)$ (av. 5.6). Macroconidia
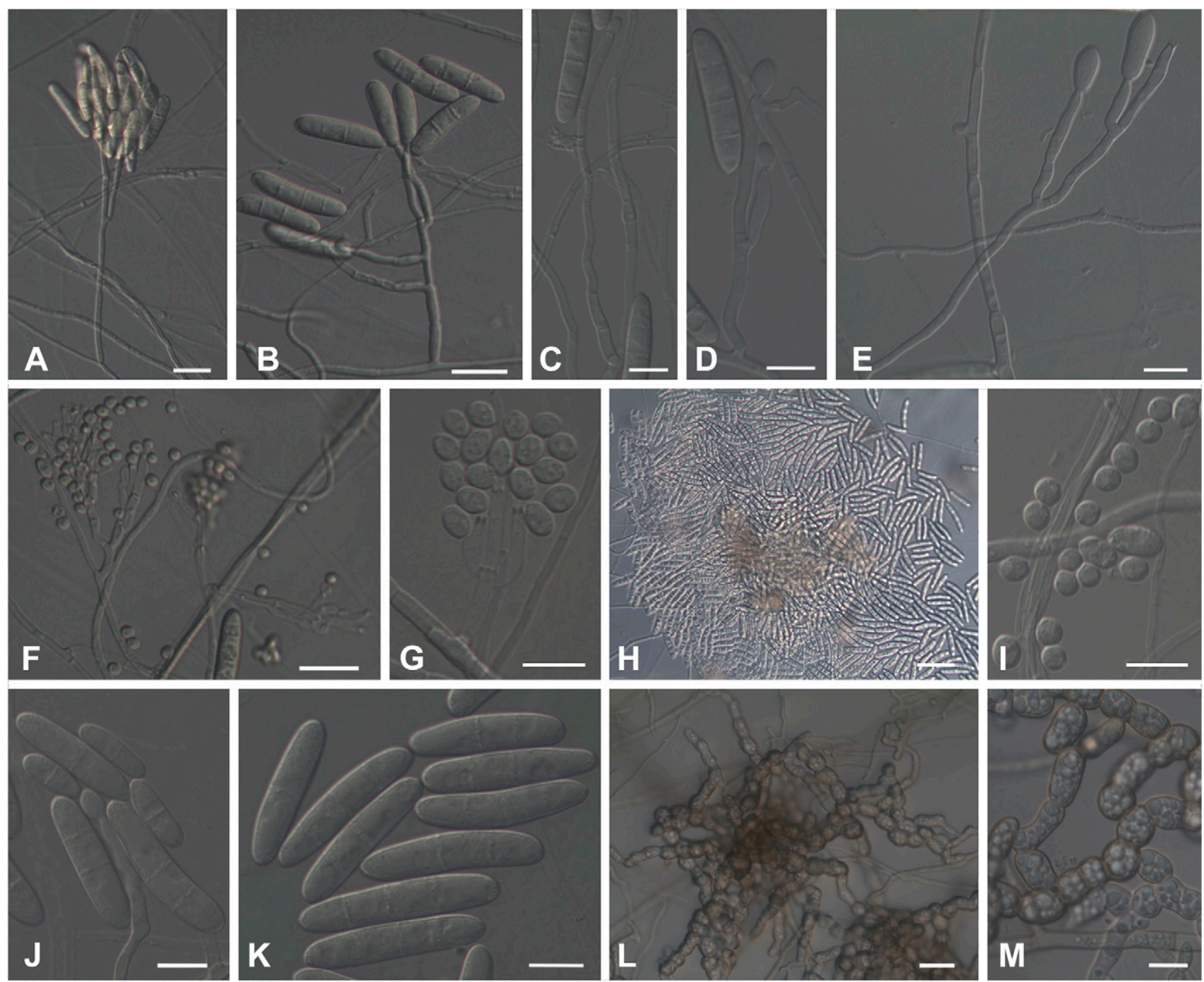

Fig. 6. Dactylonectria valentina. A-E, Simple, sparsely branched conidiophores of the aerial mycelium. E-G, Conidiophores forming microconidia arising from mycelium at agar surface, with a terminal arrangement of phialides, ranging from 2 to a dense cluster; sparsely branched or penicillate. $\mathrm{H}$, Sporodochial conidiophores. I-K, Micro- and macroconidia. L-M, Chlamydospores in mycelium. Scale bars: $\mathrm{H}=50 \mu \mathrm{m} ; \mathrm{A}-\mathrm{B}, \mathrm{F}, \mathrm{L}=20 \mu \mathrm{m} ; \mathrm{C}-\mathrm{E}, \mathrm{G}, \mathrm{I}-\mathrm{K}, \mathrm{M}=10 \mu \mathrm{m}$. 
predominant, formed by both types of conidiophores, forming flat domes of slimy masses.

Microconidia aseptate to 1-septate, with a minutely or clearly laterally displaced hilum; aseptate microconidia formed in simple conidiphores ellipsoidal to oval to fusiform (5-) 9 to $9.5(-14.5) \times(2.5-)$ 3.4 to $3.6(-5) \mu \mathrm{m}$ (av. $9.3 \times 3.5 \mu \mathrm{m}$ ), L/W ratio (1.5-) 2.6 to 2.8 (-4.3) (av. 2.7); aseptate microconidia globose to subglobose formed in complex conidiophores 4.5 to $6 \times 4$ to $4.5 \mu \mathrm{m}$; 1 -septate microconidia fusiform to ellipsoidal, (12-) 15.5 to $16.5(-20) \times(3.5-) 4.3$ to $4.6(-5.5) \mu \mathrm{m}$ (av. $16 \times 4.5 \mu \mathrm{m}), \mathrm{L} / \mathrm{W}$ ratio $(2.5-) 3.5$ to $3.8(-4.5)$ (av. 3.7); microconidia formed in heads on simple conidiophores or as masses on complex conidiophores.

Chlamydospores globose to subglobose, 11 to $20 \times 10$ to $19 \mu \mathrm{m}$ diam., smooth, but often appearing rough due to deposits, thickwalled, formed in lateral branches, rarely intercalary, mostly isolated, hyaline, becoming medium brown.

Culture characteristics: Mycelium felty with average density. Surface on OA fawn to cinnamon with aerial mycelium dark buff, with a buff margin. On PDA, sepia with aerial mycelium, vinaceous buff, and margin buff. Zonation absent, with homogeneous transparency and margins even (OA) or lobate (PDA). Colonies similar in reverse, except in color, grayish sepia (OA) and dark brick to sepia (PDA). Colonies on PDA grow 6 to $7 \mathrm{~mm}$ diameter at $5^{\circ} \mathrm{C}$ after 7 days. Optimum temperature at $25^{\circ} \mathrm{C}$, when colonies reach 62 to $64 \mathrm{~mm}$ diameter after 7 days. Colony diameter was 13 to $16 \mathrm{~mm}$ at $30^{\circ} \mathrm{C}$ after 7 days. No growth was observed at $35^{\circ} \mathrm{C}$.

Additional cultures examined: $\mathrm{Cy}-\mathrm{FO}-224$ and Cy-FO-226. Spain:

Tarragona, isolated from Ilex sp. roots, 2012, B. Mora-Sala.

Host and distribution: Ilex sp. (roots) (Spain, Tarragona).

Notes: I. ilicicola is phylogenetically closely related to I. protearum, I. leucospermi, and I. cyclaminicola based on the phylogenetic inference in this study. The morphology of these four species overlap. I. ilicicola, I. protearum, and I. cyclaminicola formed sporodochia within 5 weeks, and have solitary chlamydospores and can be distinguished from I. leucospermi that failed to form sporodochia after 8 weeks incubation at $24^{\circ} \mathrm{C}$ under continuous UV light and have intercalary chlamydospores (Cabral et al. 2012a; Lombard et. al. 2013).

N. quercicola B. Mora-Sala, A. Cabral, J. Armengol \& P. AbadCampos, sp. nov. MycoBank MB 823852 (Fig. 8). Etymology: Name refers to the plant host genus, Quercus, from which this fungus was isolated.

Diagnosis: $N$. quercicola can be distinguish morphologically by having long conidiophores that terminate in a whorl of phialides, and for production only 1-septate macroconidia. Phylogenetically it is better distinguished with the genes his 3 and tefl.

Typus: Spain: Alicante, Alcoi, 2011, on Q. ilex roots, P. AbadCampos (CBS H-23353 - holotype; CBS 143704 = Cy-FO-3 - extype culture.
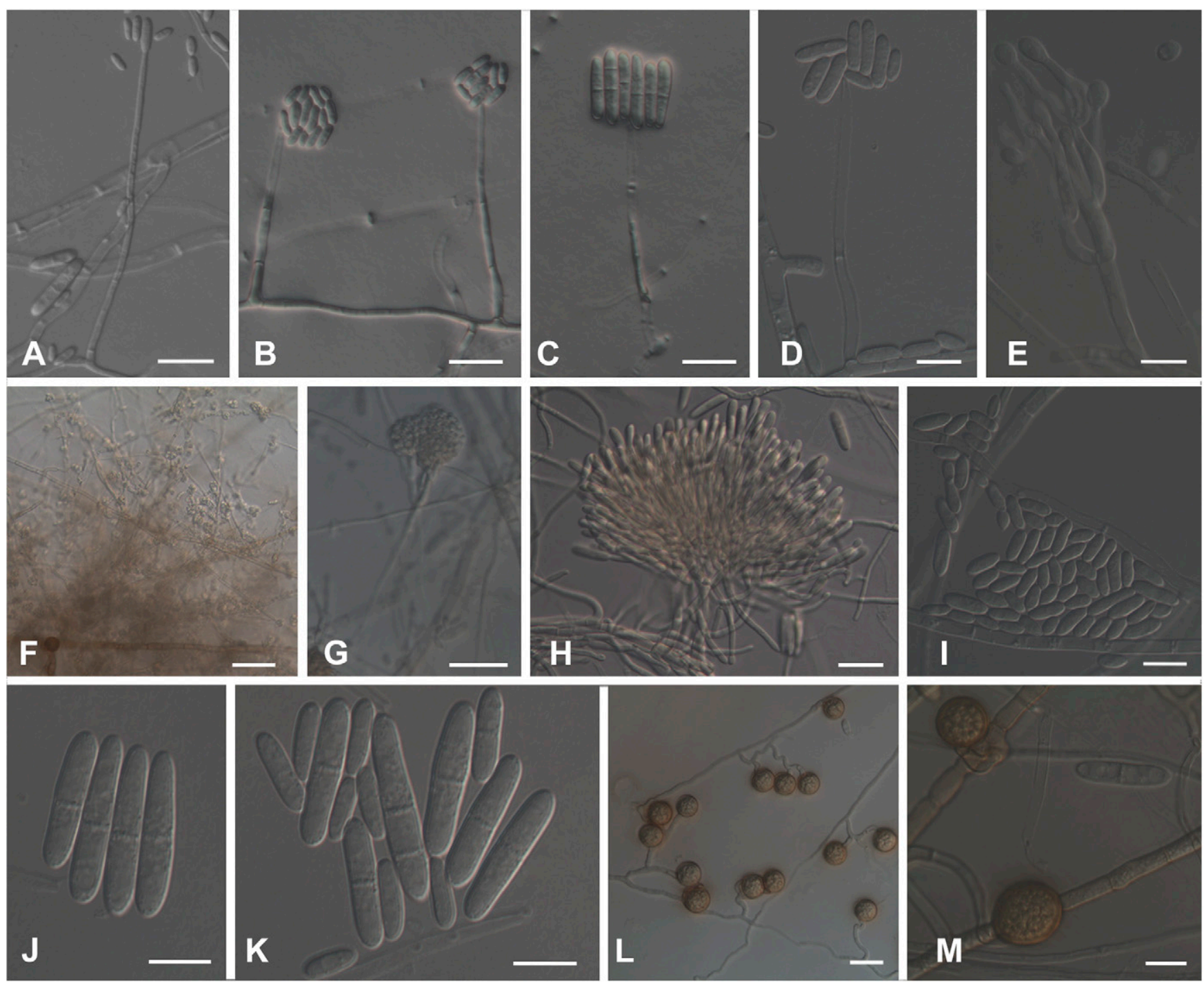

Fig. 7. Ilyonectria ilicicola (ex-type culture Cy-FO-225). A-E, Simple, sparsely branched conidiophores of the aerial mycelium. F-H, Complex conidiophores. I-K, Micro- and macroconidia. L-M, Chlamydospores in mycelium. Scale bars: F, G $=50 \mu \mathrm{m} ; A-C, L=20 \mu \mathrm{m} ; \mathrm{D}-\mathrm{E}, \mathrm{I}-\mathrm{K}, \mathrm{M}=10 \mu \mathrm{m} ; \mathrm{E}, \mathrm{H}, \mathrm{M}$ from Cy-FO-224 and A-D, F-G, I-L from CyFO-225. 
Conidiophores simple or complex. Simple conidiophores short and sparsely branched, 1 - to 2-septate, and 30 to $60 \mu \mathrm{m}$ long, or long with 4- to 7-septate, 150 to $390 \mu \mathrm{m}$ long, and terminating in a whorl of phialides; phialides monophialidic, cylindrical, tapering toward the apex, 15 to $25 \mu \mathrm{m}$ long, 1.5 to $4.0 \mu \mathrm{m}$ wide at the base, 2.0 to $4.0 \mu \mathrm{m}$ at the widest point, and 1.0 to $2.5 \mu \mathrm{m}$ near the aperture. Sporodochial conidiophores irregularly branched.
Macroconidia 1-septate, straight, cylindrical with both ends more or less broadly rounded, mostly with a visible centrally located to laterally displaced hilum; 1 -septate (17.5-) 21.5 to $22.5(-26.5) \times(4-) 4.5$ to $5(-6) \mu \mathrm{m}$ (av. $22 \times 4.7 \mu \mathrm{m}$ ), L/W ratio (3.5-) 4.6 to 4.8 (-6) (av. 4.7). Macroconidia formed in heads or as flat domes of slimy masses.

Microconidia rarely formed ( 0 to) 1 -septate; 1 -septate microconidia, mostly without a visible hilum, ellipsoidal to oblong (10-) 12 to
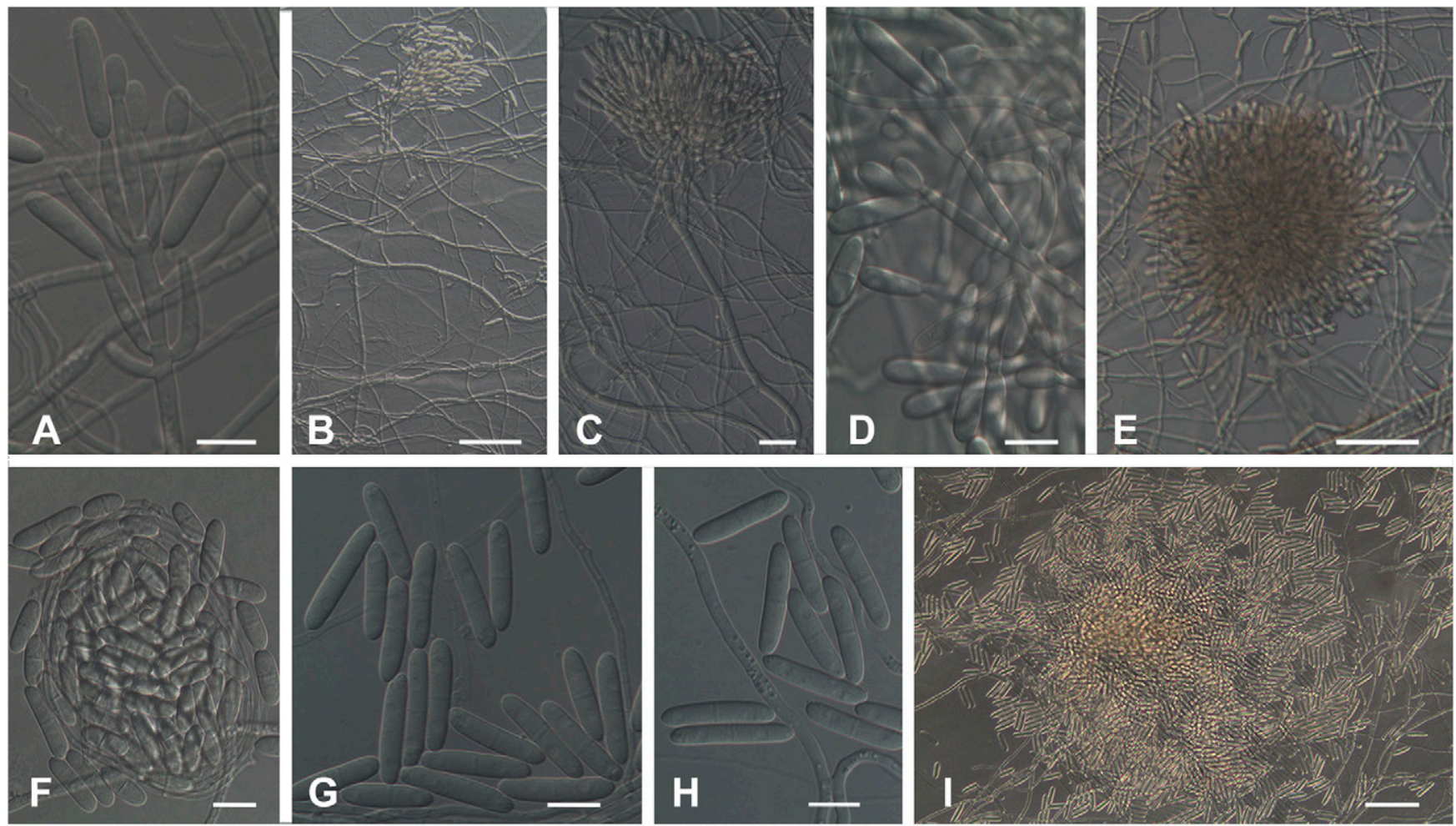

Fig. 8. Neonectria quercicola. A, Simple, sparsely branched conidiophores of the aerial mycelium. B-D, Long conidiophores of the aerial mycelium. E, Sporodochial conidiophores. F-I, Micro- and macroconidia. Scale bars: B, E, I = 50 $\mu \mathrm{m} ; \mathrm{C}=20 \mu \mathrm{m} ; \mathrm{A}, \mathrm{D}, \mathrm{F}-\mathrm{H}=10 \mu \mathrm{m}$. All from Cy-FO-3.

Table 2. Distribution of the Cylindrocarpon-like isolates according to the fungal species identified in the study. Number and percentages of isolates, hosts, and forest nurseries.

\begin{tabular}{|c|c|c|c|c|c|c|c|c|}
\hline \multirow[b]{2}{*}{ Fungal Species } & \multicolumn{2}{|c|}{ Isolates $^{\mathbf{a}}$} & \multicolumn{3}{|r|}{ Host $^{b}$} & \multicolumn{3}{|r|}{ Nurseryc } \\
\hline & No. & $\%$ & No. & $\%$ & Genera & No. & $\%$ & Location (Province) \\
\hline Cylindrodendrum alicantinum & 1 & 0.97 & 1 & 6.67 & Quercus & 1 & 5.88 & Valencia \\
\hline Dactylonectria hispanica & 1 & 0.97 & 1 & 6.67 & Pinus & 1 & 5.88 & Valencia \\
\hline D. macrodidyma & 25 & 24.27 & 9 & 60.00 & $\begin{array}{l}\text { Juniperus, Ilex, Lonicera, Myrtus, } \\
\text { Pyracantha, Quercus, Pinus, Rosmarinus }\end{array}$ & 9 & 52.94 & $\begin{array}{l}\text { Alicante, Castellón, Logroño, } \\
\text { Valencia }\end{array}$ \\
\hline D. novozelandica & 30 & 29.13 & 10 & 66.67 & $\begin{array}{l}\text { Crataegus, Juniperus, Pinus, Pistacia, } \\
\text { Quercus, Rosmarinus, Santolina }\end{array}$ & 11 & 64.71 & $\begin{array}{l}\text { Alicante, Castellón, Logroño, } \\
\text { Valencia }\end{array}$ \\
\hline D. pauciseptata & 3 & 2.91 & 2 & 13.33 & Abies, Pinus & 2 & 11.76 & Valencia, León \\
\hline D. pinicola & 1 & 0.97 & 1 & 6.67 & Abies & 1 & 5.88 & León \\
\hline D. torresensis & 12 & 11.65 & 6 & 40.00 & $\begin{array}{l}\text { Arbutus, Cistus, Juglans, Pinus, } \\
\text { Quercus, Rosmarinus }\end{array}$ & 6 & 35.29 & Alicante, Castellón, Soria, Valencia \\
\hline D. valentina & 1 & 0.97 & 1 & 6.67 & Ilex & 1 & 5.88 & Valencia \\
\hline Ilyonectria capensis & 3 & 2.91 & 3 & 20.00 & Arbutus, Juniperus, Pinus & 3 & 17.65 & Logroño, Valencia \\
\hline Ilyonectria cyclaminicola & 1 & 0.97 & 1 & 6.67 & Quercus & 1 & 5.88 & Castellón \\
\hline Ilyonectria ilicicola & 3 & 2.91 & 1 & 6.67 & Ilex & 1 & 5.88 & Tarragona \\
\hline Ilyonectria liriodendri & 9 & 8.74 & 5 & 33.33 & Arbutus, Juniperus, Pinus, Quercus & 6 & 35.29 & Castellón, Logroño, Valencia \\
\hline Ilyonectria pseudodestructans & 1 & 0.97 & 1 & 6.67 & Quercus & 1 & 5.88 & Valencia \\
\hline Ilyonectria robusta & 2 & 1.94 & 1 & 6.67 & Juglans & 1 & 5.88 & Soria \\
\hline Ilyonectria rufa & 9 & 8.74 & 6 & 40.00 & Abies, Arbutus, Juniperus, Quercus, Pinus & 6 & 35.29 & Alicante, Castellón, León, Valencia \\
\hline Neonectria quercicola & 1 & 0.97 & 1 & 6.67 & Quercus & 1 & 5.88 & Alicante \\
\hline
\end{tabular}

\footnotetext{
a Number of isolates and percentages were calculated on the basis of a total of 103 isolates.

${ }^{b}$ Number of hosts and percentages were calculated on the basis of a total of 15 plant genera.

${ }^{c}$ Number of nurseries and percentages were calculated on the basis of a total of 17 surveyed nurseries.
} 
$13.5(-15) \times(4-) 4.5$ to $5(-5.5) \mu \mathrm{m}($ av. $13 \times 4.8 \mu \mathrm{m}), \mathrm{L} / \mathrm{W}$ ratio $(2-)$ 2.5 to $3(-3.5)$ (av. 2.7).

Chlamydospores not observed.

Culture characteristics: Mycelium felty with low density (OA) or strong density (PDA). Surface on OA buff with aerial mycelium cinnamon. Surface on PDA pale buff; with a pale luteous concentric ring. Zonation absent (OA) to concentric (PDA), transparency homogeneous and margins even. Reverse similar to surface, except in color, light cinnamon (OA) and buff with center sepia (PDA).

Colonies on PDA grow $5.7 \mathrm{~mm}$ diameter at $5^{\circ} \mathrm{C}$ after 7 days. Optimum temperature at $25^{\circ} \mathrm{C}$, when colonies reach $17.2 \mathrm{~mm}$ diameter, after 7 days. Colony diameter was $6.4 \mathrm{~mm}$ at $30^{\circ} \mathrm{C}$ after 7 days. No growth was observed at $35^{\circ} \mathrm{C}$.

Host and distribution: $Q$. ilex (Spain, Alicante, Alcoi).

Notes: Based on the phylogenetic inference in this study, N. quercicola is closely related to other three isolates with no description available (CPC 13530, CPC 13531 and CR21). These isolates should also be considered $N$. quercicola, as they form a clade very well supported with $100 \%$ bootstrap support and a Bayesian posterior probability of 1.0.

Host distribution. The fungal species identified in this study were found associated with 15 host genera: Abies (2.9\%), Arbutus $(5.8 \%)$, Cistus (1.9\%), Crataegus (1\%), Ilex (4.9\%), Juglans (2.9\%), Juniperus (6.8\%), Lonicera (1\%), Myrtus (1\%), Pinus (37.9\%), Pistacia (1.9\%), Pyracantha (1\%), Quercus (26.2\%), Rosmarinus (3.9\%), and Santolina (1\%) (Table 2). Pinus was the genus with the highest number of isolates recovered and from which eight species were identified: $D$. hispanica, D. macrodidyma, D. novozelandica, D. pauciseptata, D. torresensis, I. capensis, I. liriodendri, and I. rufa. Quercus was the only host from which the four fungal genera were isolated, and the host with the highest number of fungal species isolated: D. macrodidyma, D. novozelandica, D. torresensis, I. cyclaminicola, I. liriodendri, I. pseudodestructans, I. rufa, C. alicantinum, and $N$. quercicola. The third host regarding the number of species isolated was Juniperus: D. macrodidyma, D. novozelandica, D. capensis, I. liriodendri, and I. rufa, followed by Arbutus, from which four fungal species were recovered, and Abies and Rosmarinus, from which three fungal species were recovered. In Ilex and Juglans, only two fungal species were recovered and only one from Cistus, Crataegus, Lonicera, Myrtus, Pistacia, Pyracantha, and Santolina.

Regarding the species, D. novozelandica was recovered from $66.67 \%$ of the hosts, followed by D. macrodidyma $(60 \%), D$. torresensis and I. rufa (40\%), I. liriodendri (33.33\%), I. capensis (20\%), and D. pauciseptata (13.33\%). The remaining fungal species were only recovered from one host, which represented $6.67 \%$ of the total number of hosts surveyed in this study (Table 2).

\section{Discussion}

The present study represents the first attempt to characterize a wide collection of Cylindrocarpon-like asexual morphs collected from forest nurseries in Spain. This clearly demonstrates the prevalence of this fungal group associated with seedlings of diverse number of hosts showing decline symptoms. Cylindrocarpon-like asexual morphs are ubiquitous and can be easily found in soil or associated with plant roots, some of them also having a potential role as latent pathogens or endophytic organisms (Agustí-Brisach and Armengol 2013; Halleen et al. 2006).

Sixteen species belonging to the genera Cylindrodendrum, Dactylonectria, Ilyonectria, and Neonectria were identified from damaged roots of 15 forest plant genera. Six isolates were not identified to the species level with the his 3 data. Although the his3 region has previously shown to be a very informative locus (Cabral et al. 2012a), a combined analysis with ITS, tub2, and tef1 regions better resolved and confirmed that these isolates represented novel phylogenetic species, newly described as $D$. hispanica, $D$. valentina. I. ilicicola, and N. quercicola.

This is the first report of C. alicantinum on Q. ilex; of D. macrodydima on Ilex aquifolium, Juniperus phoenicea, Lonicera sp., Myrtus communis, P. halepensis, Pyracantha sp., Q. faginea, $Q$. ilex, and Rosmarinus officinalis; of D. novozelandica on Crataegus azarolus, J. phoenicea, Pinus sp., P. halepensis, Pistacia lentiscus, Quercus sp., Q. ilex, Q. suber, R. officinalis, and Santolina chamaecyparissus; of D. pauciseptata on Abies nordmanniana and P. halepensis; of D. pinicola on A. concolor, of D. torresensis on Ar. unedo, Cistus albidus, Ju. regia, P. halepensis, Q. ilex, and R. officinalis; of I. capensis in Arbutus unedo, Juniperus sp., and P. halepensis; of I. cyclaminicola on Quercus sp.; of I. liriodendri on Ar. unedo, Juniperus sp., $P$. halepensis; of I. pseudodestructans on $Q$. ilex; of I. robusta on Ju. regia; and of I. rufa on A. nordmanniana, Ar. unedo, Juniperus sp., $P$. halepensis, $Q$. faginea, and $Q$. ilex. Furthermore, this is the first report of I. capensis in Europe because, to our knowledge, this fungus had only been recorded affecting Protea in South Africa (Lombard et al. 2013).

To date, D. novozelandica, D. macrodidyma, D. torresensis, I. liriodendri, I. robusta, and C. alicantinum had been reported only in cultivated crops such as grapevine (Vitis vinifera) (Agustí-Brisach and Armengol 2013) or loquat (Eriobotrya japonica) (AgustíBrisach et al. 2016) in Spain, but never affecting forest plants.

D. pauciseptata had been reported as a pathogen of grapevines in Slovenia and New Zealand (Schroers et al. 2008), Uruguay (Abreo et al. 2010), Spain (Martín et al. 2011), Portugal (Cabral et al. 2012a), Brazil (dos Santos et al. 2014), and British Columbia, Canada (Úrbez-Torres et al. 2014). It has also been reported as a pathogen of apple trees in South Africa and of peach trees in Italy (Tewoldemedhin et al. 2011; Yaseen et al. 2012). This fungus had also been recorded in forest hosts Viburnum tinus in Italy (Aiello et al. 2015) and $P$. radiata in Spain (Agustí-Brisach et al. 2011). Thus, this study increases the range of forest hosts in nursery for this species, representing the first report of D. pauciseptata on A. nordmanniana and $P$. halepensis.

In our study, no correlation was found between the fungal pathogens isolated and nurseries from which they were collected. The fungal species did not show any distribution pattern among the different locations surveyed, perhaps because of the small sample size, as three plants per host is probably not enough to look for these correlations. Furthermore, it should be taken into account that there were locations (provinces) in which a higher number of nurseries were surveyed, including Valencia, Castellón, and Alicante. Likewise, some of the surveyed hosts, in particular Pinus and Quercus, were the target hosts of the survey undertaken due to the importance of these genera in Spanish forests (MAGRAMA 2014). Therefore, these hosts had a higher number of sampled plants, also corresponding with a higher number of Cylindrocarpon-like isolates compared with other hosts. These two genera prevail in the Mediterranean landscape, as they constitute the characteristic vegetation of the Mediterranean forests. In this regard, the presence of nine Cylindrocarpon-like species on Quercus and eight on Pinus trees highlight the need for better management of nursery diseases to avoid the dispersal of these fungi through planting materials used for reforestation purposes. Management of Cylindrocarpon-like asexual morphs associated with black-foot disease has been intensively studied on grapevine nurseries, where the incorporation of holistic and integrated control measures such as cultural practices, sanitation, and chemical and biological control has been shown as the best approach to improve the phytosanitary quality of planting material (Gramaje and Armengol 2011; Gramaje et al. 2018). Implementing a similar strategy could be advisable in forest nurseries.

\section{Acknowledgments}

We would like to thank E. Pérez-Laorga from Conselleria de Agricultura, Medio Ambiente, Cambio Climático y Desarrollo Rural (Generalitat Valenciana), who facilitated nursery surveys. We would like to thank M. Ferrer for his technical assistance.

\section{Literature Cited}

Abreo, E., Martinez, S., Bettucci, L., and Lupo, S. 2010. A morphological and molecular characterization of Campylocarpon and Cylindrocarpon spp. associated with black foot disease of grapevines in Uruguay. Australas. Plant Pathol. 39:446-452.

Agustí-Brisach, C., and Armengol, J. 2013. Black-foot disease of grapevine: an update on taxonomy, epidemiology and management strategies. Phytopathol. Mediterr. 52:245-261. 
Agustí-Brisach, C., Cabral, A., González-Domínguez, E., Pérez-Sierra, A., León, M., Abad-Campos, P., García-Jiménez, J., Oliveira, H., and Armengol, J. 2016. Characterization of Cylindrodendrum, Dactylonectria and Ilyonectria isolates associated with loquat decline in Spain, with description of Cylindrodendrum alicantinum sp. nov. Eur. J. Plant Pathol. 145:103-118.

Agustí-Brisach, C., Pérez-Sierra, A., García-Figueres, F., Montón, C., and Armengol, J. 2011. First report of damping-off caused by Cylindrocarpon pauciseptatum on Pinus radiata in Spain. Plant Pathol. 95:874.

Aiello, D., Guarnaccia, V., Epifani, F., Perrone, G., and Polizzi, G. 2015. 'Cylindrocarpon' and Ilyonectria species causing root and crown rot disease of potted Laurustinus plants in Italy. J. Phytopathol. 163:675-680.

Axelrood, P. E., Chapman, W. K., Seifert, K. A., Trotter, D. B., and Shrimpton, G. 1998. Cylindrocarpon and Fusarium root colonization of Douglas-fir seedlings from British Columbia reforestation sites. Can. J. For. Res. 28:1198-1206.

Beyer-Ericson, L., Damm, E., and Unestam, T. 1991. An overview of root dieback and its causes in Swedish forest nurseries. Eur. J. Forest Pathol. 21:439-443.

Booth, C. 1966. The genus Cylindrocarpon. Mycol. Pap. 104:1-56.

Brasier, C. M. 2008. The biosecurity threat to the UK and global environment from international trade in plants. Plant Pathol. 57:792-808.

Brayford, D. 1993. Cylindrocarpon. Pages 103-106 in: Methods for Research on Soilborne Phytopathogenic Fungi. L. L. Singleton, J. D. Mihail, and C. M. Rush, eds. American Phytopathological Society Press, St. Paul, MN.

Brayford, D., Honda, B. M., Mantiri, F. R., and Samuels, G. J. 2004. Neonectria and Cylindrocarpon: the Nectria mammoidea group and species lacking microconidia. Mycologia 65:256-261

Cabral, A., Groenewald, J. Z., Rego, C., Oliveira, H., and Crous, P. W. 2012a. Cylindrocarpon root rot: multi-gene analysis reveals novel species within the Ilyonectria radicicola species complex. Mycol. Prog. 11:655-688.

Cabral, A., Rego, C., Nascimento, T., Oliveira, H., Groenewald, J. Z., and Crous, P. W. 2012b. Multi-gene analysis and morphology reveal novel Ilyonectria species associated with black foot disease of grapevines. Fungal Biol-UK 116:62-80.

Castlebury, L. A., Rossman, A. Y., and Hyten, A. S. 2006. Phylogenetic relationships of Neonectria/Cylindrocarpon on Fagus in North America. Can. J. Bot. 84:1417-1433.

Chaverri, P., Salgado, C., Hirooka, Y., Rossman, A. Y., and Samuels, G. J. 2011. Delimitation of Neonectria and Cylindrocarpon (Nectriaceae, Hypocreales, Ascomycota) and related genera with Cylindrocarpon-like anamorphs. Stud. Mycol. 68:57-78.

Crous, P. W., Gams, W., Stalpers, J. A., Robert, V., and Stegehuis, G. 2004a. MycoBank: an online initiative to launch mycology into the 21st century. Stud. Mycol. 50:19-22.

Crous, P. W., Groenewald, J. Z., Risede, J. M., and Hywel-Jones, N. 2004b. Calonectria species and their Cylindrocladium anamorphs: species with sphaeropedunculate vesicles. Stud. Mycol. 50:415-429.

Crous, P. W., Verkley, G. J. M., Groenewald, J. Z., and Samson, R. A. 2009. CBS Laboratory Manual Series 1: Fungal Biodiversity. CBS-KNAW Fungal Biodiversity Centre, Utrecht, The Netherlands.

Darriba, D., Taboada, G. L., Doallo, R., and Posada, D. 2012. jModelTest 2: more models, new heuristics and parallel computing. Nat. Methods 9:772.

Dhingra, O. D., and Sinclair, J. B. 1995. Basic Plant Pathology Methods, 2nd Ed. CRC Press, Boca Ratón, FL.

dos Santos, R. F., Blume, E., Muniz, M. F. B., Steckling, S. M., Burtet, G. W., Harakawa, R., Garrido, L. R., and Reiniger, L. R. S. 2014. First report of "Cylindrocarpon" pauciseptatum associated with black foot disease of grapevine in Brazil. Plant Dis. 98:567.

Dumroese, R. K., and James, R. L. 2005. Root diseases in bareroot and container nurseries of the Pacific Northwest: epidemiology, management, and effects on outplanting performance. New For. 30:185-202.

FAO. 2015. Global forests resources assessment 2015. ISBN 978-92-5-108826-5. Rome, Italy.

Gardes, M., and Bruns, T. D. 1993. ITS primers with enhanced specificity for basiodiomycetes-applications to the identification of mycorrhizae and rusts. Mol. Ecol. 2:113-118.

Glass, N. L., and Donaldson, G. 1995. Development of primer sets designed for use with PCR to amplify conserved genes from filamentous ascomycetes. Appl. Environ. Microbiol. 61:1323-1330.

Gramaje, D., and Armengol, J. 2011. Fungal trunk pathogens in the grapevine propagation process: potential inoculum sources, detection, identification, and management strategies. Plant Dis. 95:1040-1055.

Gramaje, D., Úrbez-Torres, J. R., and Sosnowski, M. R. 2018. Managing grapevine trunk diseases with respect to etiology and epidemiology: current strategies and future prospects. Plant Dis. 102:12-39.

Halleen, F., Fourie, P. H., and Crous, P. W. 2006. A review of black foot disease of grapevine. Phytopathol. Mediterr. 45:55-67.

Halleen, F., Schroers, H. J., Groenewald, J. Z., and Crous, P. W. 2004. Novel species of Cylindrocarpon (Neonectria) and Campylocarpon gen. nov. associated with black-foot disease of grapevines (Vitis spp.). Stud. Mycol. 50:431-455.

Halmschlager, E., and Kowalski, T. 2004. The mycobiota in nonmycorrhizal roots of healthy and declining oaks. Can. J. Bot. 82:1446-1458.

Hirooka, Y., Ichihara, Y., Masuya, H., and Kubono, T. 2012. Seed rot, a new disease of beech tree caused by Neonectria ramulariae (anamorph: Cylindrocarpon obtusiusculum). J. Phytopathol. 160:504-506.
Iles, T. M., Ashton, D. H., Kelliher, K. J., and Keane, P. J. 2010. The effect of Cylindrocarpon destructans on the growth of Eucalyptus regnans seedlings in air-dried and undried forest soil. Aust. J. Bot. 58:133-140.

Jankowiak, R., Stepniewska, H., Szwagrzyk, J., Bilánski, P., and Gratzer, G. 2016. Characterization of Cylindrocarpon-like species associated with litter in the old-growth beech forests of Central Europe. For. Pathol. 46:582-594.

Kowalski, S. 1982. Role of mycorrhiza and soil fungi in natural regeneration of fir (Abies alba Mill.) in Polish Carpathians and Sudetes. Eur. J. Forest Pathol. 12: $107-112$

Krzan, Z. 1987. Fungi inhabiting roots of seedlings in two selected stands in Tatrzanski National Park. Parki Nar. Rezerw. Przyr. 8:5-14 (in Polish).

Kubíková, J. 1963. The surface mycoflora of ash roots. Trans. Br. Mycol. Soc. 46: 107-114.

Kumar, S., Stecher, G., and Tamura, K. 2016. MEGA7: Molecular Evolutionary Genetics Analysis version 7.0 for bigger datasets. Mol. Biol. Evol. 33 1870-1874.

Lilja, A., Lilja, S., Poteri, M., and Ziren, L. 1992. Conifer seedling root fungi and root dieback in Finnish nurseries. Scand. J. For. Res. 7:547-556.

Lombard, L., Bezuidenhout, C. M., and Crous, P. W. 2013. Ilyonectria black foot rot associated with Proteaceae. Australas. Plant Pathol. 42:337-349.

Lombard, L., Van Der Merwe, N. A., Groenewald, J. Z., and Crous, P. W. 2014. Lineages in Nectriaceae: Re-evaluating the generic status of Ilyonectria and allied genera. Phytopathol. Mediterr. 53:340-357.

MAGRAMA. 2014. Análisis y Prospectiva. Diagnóstico del Sector Forestal Español. Serie Agrinfo/Medioambiente $\mathrm{n}^{\circ}$ 8. Ministerio de Agricultura, Alimentación y Medio Ambiente, Madrid, Spain. https://www.mapama.gob. es/es/ministerio/servicios/analisis-y-prospectiva/AyP_serie\%20n\%C2\%BA8\% 20diagn\%C3\%B3stico\%20sector\%20FORESTAL_tcm30-88409.pdf

Manka, K., Gierczak, M., and Prusinkiewicz, Z. 1968. The succumbing of yew-tree seedlings in Wierzchlas and the saprophytic fungi of the soil environment. Prace Komisji Nauk Rol. Lesnych 25:177-195 (in Polish).

Mantiri, F. R., Samuels, G. J., Rahe, G. E., and Honda, B. 2001. Phylogenetic relationships in Neonectria species having Cylindrocarpon anamorphs inferred from mitochondrial ribosomal DNA sequences. Can. J. Bot. 79: 334-340.

Martín, M. T., Martín, L., Cuesta, M. J., and García-Benavides, P. 2011. First report of Cylindrocarpon pauciseptatum associated with grapevine decline from Castilla y León, Spain. Plant Dis. 95:361.

Menkis, A., Vasiliauskas, R., Taylor, A. F. S., Stenström, E., Stenlid, J., and Finlay, R. 2006. Fungi in decayed roots of conifer seedlings from forest nurseries, afforested clearcuts and abandoned farmland. Plant Pathol. 55: $117-129$.

Miller, M. A., Pfeiffer, W., and Schwartz, T. 2010. Creating the CIPRES Science Gateway for inference of large phylogenetic trees. Pages 4-52 in: Proceedings of the Gateway Computing Environments Workshop (GCE). New Orleans, LA USA.

Mwanza, E. J. M., and Kellas, J. D. 1987. Identification of the fungi associated with damping-off in the regeneration of Eucalyptus obliqua and E. radiata in a central Victorian forest. Eur. J. Forest Pathol. 17:237-245.

Nirenberg, H. 1976. Untersuchungen über die morphologische und biologische Differenzierung in der Fusarium-Section Liseola. Mitteilungen aus der Biologischen Bundesanstalt für Land- und Forstwirtschaft 169:1-117.

O'Donnell, K., and Cigelnik, E. 1997. Two divergent intragenomic rDNA ITS2 types within a monophyletic lineage of the fungus Fusarium are nonorthologous. Mol. Phylogenet. Evol. 7:103-116.

Rayner, R. W. 1970. A Mycological Colour Chart. Commonwealth Mycological Institute, Kew, Surrey, UK.

Robinson, O., Dylus, D., and Dessimoz, C. 2016. Phylo.io: Interactive viewing and comparison of large phylogenetic trees on the web. Mol. Biol. Evol. 33: 2163-2166

Rodríguez, F., Oliver, J. F., Marin, A., and Medina, J. R. 1990. The general stochastic model of nucleotide substitutions. J. Theor. Biol. 142:485-501.

Ronquist, F., Teslenko, M., van der Mark, P., Ayers, D. L., Darling, A., Höhna, S., Larget, B., Liu, L., Suchard, M. A., and Huelsenbeck, J. P. 2012. MrBayes 3.2: efficient Bayesian phylogenetic inference and model choice across a large modelspace. Syst. Biol. 61:539-542.

Rossman, A. Y., Samuels, G. J., Rogerson, C. T., and Lowen, R. 1999. Genera of the Bionectriaceae, Hypocreaceae, and Nectriaceae (Hypocreales, Ascomycetes). Stud. Mycol. 42:1-248.

Sánchez, M. E., Lora, F., and Trapero, J. A. 2002. First report of Cylindrocarpon destructans as a root pathogen of Mediterranean Quercus species in Spain. Plant Dis. 86:693.

Schroers, H. J., Žerjav, M., Munda, A., Halleen, F., and Crous, P. W. 2008. Cylindrocarpon pauciseptatum sp. nov., with notes on Cylindrocarpon species with wide, predominantly 3-septate macroconidia. Mycol. Res. 112: $82-92$

Szewczyk, J., and Szwagrzyk, J. 2010. Spatial and temporal variability of natural regeneration in a temperate old-growth forest. Ann. For. Sci. 67:202.

Tewoldemedhin, Y. T., Mazzola, M., Mostert, L., and McLeod, A. 2011 Cylindrocarpon species associated with apple tree roots in South Africa and their quantification using real-time PCR. Eur. J. Plant Pathol. 129:637-651.

Trumbore, S., Brando, P., and Hartmann, H. 2015. Forest health and global change. Science 349:814-818 
Úrbez-Torres, J. R., Haag, P., and O’Gorman, D. T. 2014. Grapevine trunk diseases in British Columbia: incidence and characterization of the fungal pathogens associated with esca and Petri diseases of grapevine. Plant Dis. 98:469-482.

Vaidya, G., Lohman, D. J., and Meier, R. 2011. SequenceMatrix: concatenation software for the fast assembly of multigene datasets with character set and codon information. Cladistics 27:171-180.

White, T. J., Burns, T., Lee, S., and Taylor, J. W. 1990. Amplification and direct sequencing of fungal ribosomal RNA genes for phylogenetic. Pages 315-322 in:
M. A. Innis, D. H. Gelfand, J. J. Sninsky, and T. J. White, eds. PCR Protocols: A Guide to Methods and Applications. Academic Press, San Diego, CA.

Wingfield, M. J., Brockerhoff, B. D., Wingfield, B. D., and Slippers, B. 2015.

Planted forest health: The need for a global strategy. Science 349:832836.

Yaseen, T., Ahmed, Y., D'Onghia, A. M., and Digiaro, M. 2012. First report of Cylindrocarpon pauciseptatum associated with root rot and decline of peach in southern Italy (Apulia region). Plant Dis. 96:764. 